\title{
Mid-Cenozoic SHRIMP U-Pb detrital zircon ages from metasedimentary rocks in the North Patagonian Andes of Aysén, Chile
}

\author{
*Paulo Quezada1, Francisco Hervé2, 3 , Mauricio Calderón², Mark Fanning ${ }^{4}$, Robert Pankhurst ${ }^{5}$, \\ Estanislao Godoy ${ }^{6}$, Octavio Urbina ${ }^{7}$, Rodrigo Suárez
}

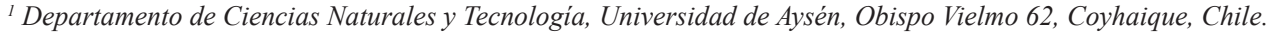 \\ p.quezada.pozo@gmail.com \\ ${ }^{2}$ Carrera de Geología, Facultad de Ingeniería, Universidad Andres Bello, Sazie 2119, Santiago. \\ fherve@unab.cl,mauricio.calderon@unab.cl \\ ${ }^{3}$ Departamento de Geología, Universidad de Chile, Plaza Ercilla 803, Santiago. \\ fherve@cec.uchile.cl \\ ${ }^{4}$ Research School of Earth Sciences, The Australian National University, Canberra, ACT 0200, Australia. \\ mark.fanning@anu.edu.au \\ ${ }^{5}$ Visiting Research Associate, British Geological Survey, Keyworth, Nottingham NG12 5GG, United Kingdom. \\ rjpankhurst@gmail.com \\ ${ }^{6}$ Tehema Consultores Geológicos, Virginia Subercaseaux 4100, Pirque, Chile. \\ egodoyster@gmail.com \\ ${ }^{7}$ Geographica, Casilla 174, Casablanca, Chile. \\ octavio.urbina2@gmail.com \\ ${ }^{8}$ Instituto de Estudios Andinos IDEAN, Universidad de Buenos Aires-CONICET, Intendente Güiraldes 2160, Ciudad Universitaria, \\ Buenos Aires, Argentina. \\ rsuarez@gl.fcen.uba.ar \\ * Corresponding author: p.quezada.pozo@gmail.com
}

\begin{abstract}
Previously undated low-grade metamorphic rocks from the Puerto Cisnes-Queulat area (44³0’ S) contain detrital zircons of mid-Oligocene age (ca. $28 \mathrm{Ma})$. Their outcrops represent the easternmost occurrence of the late Oligocene to early Miocene marine volcano-sedimentary Traiguén Formation; previous correlation with the Paleozoic metamorphic basement of this sector of the North Patagonian Andes is thus refuted. A similar age and provenance were obtained for a paraconglomerate bed of the La Junta Formation $c a .80 \mathrm{~km}$ to the north, which is thought to represent a high-energy lateral facies variation of the Traiguén Formation. Miocene plutonic rocks of the North Patagonian Batholith intruded these metasedimentary rocks, generating a contact metamorphic aureole that reaches biotite grade and overprints a previous metamorphic fabric probably formed during closure of the Traiguén Basin. Similar young ages for metamorphic rocks located immediately west of the Liquiñe-Ofqui Fault Zone $300 \mathrm{~km}$ north, near Ayacara, suggest a regional pattern of earliest Neogene metamorphism and rapid exhumation in this segment of the Patagonian Andes.
\end{abstract}

Keywords: Traiguén Formation, Liquiñe-Ofqui Fault Zone.

RESUMEN. Edades U-Pb SHRIMP del Cenozoico medio en circones detríticos de rocas metasedimentarias en los Andes Norpatagónicos de Aysén, Chile. Rocas metamórficas de bajo grado presentes en el área Puerto CisnesQueulat (4430'S) contienen circones detríticos de edad Oligoceno medio (ca. $28 \mathrm{Ma}$ ). Estos afloramientos corresponden a la ocurrencia más oriental de las rocas volcano-sedimentarias de la Formación Traiguén, de edad Oligoceno tardío a Mioceno temprano; correlaciones previas con el basamento metamórfico de edad Paleozoica de esta porción de los 
Andes Norpatagónicos son descartadas. Una edad máxima y fuentes de procedencia similares se obtuvieron en un paraconglomerado de la Formación La Junta, unos $80 \mathrm{~km}$ al norte, el cual se interpreta como una variación lateral de alta energía de la Formación Traiguén. Rocas plutónicas del Mioceno pertenecientes al Batolito Norpatagónico intruyen a las rocas metasedimentarias, generan una aureola de contacto que alcanza el grado metamórfico de la biotita, y se sobrepone a una fábrica metamórfica anterior probablemente relacionada con el tectonismo producto del cierre de la cuenca Traiguén. Edades Cenozoicas similares han sido obtenidas para rocas metamórficas ubicadas inmediatamente al oeste de la Zona de Falla Liquiñe-Ofqui $300 \mathrm{~km}$ al norte, cerca de Ayacara, lo cual sugiere un patrón regional de metamorfismo y exhumación en una rápida sucesión durante el Neógeno más temprano en este segmento de los Andes Patagónicos.

Palabras clave: Formación Traiguén, Zona de Falla Liquiñe-Ofqui.

\section{Introduction}

Evidence of the Cenozoic evolution of the North Patagonian Andes margin is partially preserved in the western slope of the Andean Cordillera (Fig. 1). It underwent a major Neogene phase of batholith construction (Pankhurst et al., 1999) and large-scale denudation caused by transpressional tectonics (Thomson, 2002). Deformation and metamorphism were focused along the Liquiñe-Ofqui Fault Zone (LOFZ; Fig. 1), a right lateral strike-slip structure which extends for $1,000 \mathrm{~km}$ north of the Golfo de Penas $\left(c a .47^{\circ} \mathrm{S}\right)$ through the western slope of the main cordillera (Hervé, 1976, 1994; Beck et al., 1993; Cembrano et al., 1996; Cembrano et al., 2002). The early Neogene contraction resulted in the uplift of the North Patagonian Andes and development of Miocene foreland basins.

Prior to this Neogene uplift, the Andean and extraAndean areas of Patagonia were largely covered by marine deposits with Pacific and Atlantic affinities, respectively (e.g., Malumián and Yañez, 2011; Encinas et al., 2013; Bechis et al., 2014; Encinas et al., 2016a; Cuitiño et al., 2017), in some areas possibly with transient oceanic connection (see below). These Eocene-early Miocene marine strata (Fig.1) represent the climax and final stage of an extensional episode that started during the early Cenozoic (Aragón et al., 2011; Hervé et al., 2017; Encinas et al., 2019), following Lower and Upper Cretaceous orogenic phases (Gianni et al., 2018 and references therein).

At $c a .40-46^{\circ} \mathrm{S}$, the early mid-Cenozoic deepmarine strata cropping out in the western slope of the Andean Cordillera are spatially related to the main trace and secondary branches of the LOFZ (Figs. 1 y 2). The greatest extension is recorded in the Traiguén Formation (Espinoza and Fuenzalida, 1971), in which deep-marine turbidite deposits (Encinas et al., 2016a) are found overlying or associated with supra-subduction zone pillow lavas overprinted by ocean-floor metamorphism (Hervé et al., 1995). In some localities, the volcano-sedimentary units are profusely intruded by mafic dykes (Silva et al., 2003), resembling the emplacement of a dyke swarm on a thin and attenuated ocean-like crust.

In this work, we present SHRIMP U-Pb detrital zircon ages for intercalated packages of metapelite and metasandstone in the Puerto Cisnes-Queulat area (ca. $44^{\circ} 30^{\prime} \mathrm{S}$ ), east of the main trace of the LOFZ and for a paraconglomerate sample from La Junta area. These have been previously interpreted as either Paleozoic metamorphic basement (SERNAGEOMIN, 2003) or Cenozoic clastic successions (Bobenrieth et al., 1983). The results and geological considerations presented here provide constraints on their ages of deposition and metamorphism and their tectonic significance in the context of regional mid-Cenozoic evolution of the area.

\section{Regional tectonic and geological background}

According to Cenozoic plate tectonic reconstructions based on ocean floor magnetic anomalies, the convergence between Farallon/Nazca and South America plates evolved from a low-velocity oblique subduction system during the Paleogene to a faster and nearly orthogonal subduction by late Oligocene times (e.g., Cande and Leslie, 1986; Pardo-Casas and Molnar, 1987; Somoza and Ghidella, 2005; Eagles and Scott, 2014). This change reflects the birth of the Nazca plate and rearrangement of oceanic plate boundaries in east Pacific (Lonsdale, 2005; Somoza and Ghidella, 2012).

Early-mid Paleogene syn-extensional flare-up magmatism (Rapela et al., 1988) and uplift is recorded 


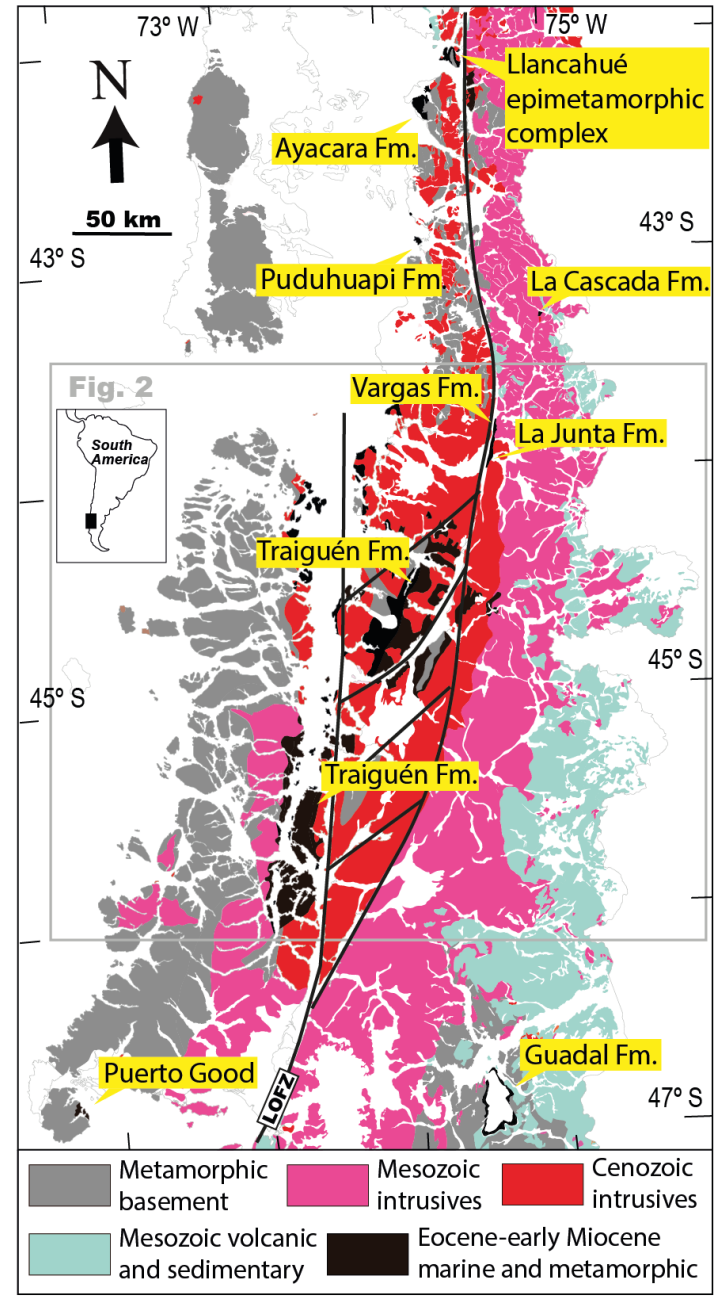

FIG. 1. Sketch map showing distribution of main geological units of Chilean Patagonia between $42^{\circ}-47^{\circ} \mathrm{S}$ (modified from SERNAGEOMIN, 2003). LOFZ: Liquiñe-Ofqui Fault Zone (fault trace after Cembrano et al., 2000).

in the western sectors of the North Patagonian Massif (Aragón et al., 2013), while more restricted plutonism is found near the LOFZ (Pankhurst et al., 1999; Duhart, 2008). During this time, the subduction and southward migration of the Aluk-Farallon seismic ridge triggered the development of a slab window (Kay et al., 2004; Espinoza et al., 2005) beneath the transform margin of northern Patagonia (Aragón et al., 2011). Widespread Oligocene magmatism then erupted in the present forearc (the Coastal Magmatic Belt of Muñoz et al., 2000) and retroarc (El Maitén Belt and Somuncurá Plateau, Rapela et al., 1988; Kay et al., 2004; Fernández Paz et al., 2019). This magmatic phase partly predates the late Oligocene to early Miocene Pacific and Atlantic marine ingressions that covered wide areas of southern South America, with possible areas of transient ocean connection (e.g., Encinas et al., 2014; Bechis et al., 2014 and references therein). In the western slope of the North Patagonian Andes, deep-marine sedimentation is mostly recorded in the Ayacara $\left(c a .42^{\circ} \mathrm{S}\right)$ and Traiguén ( $c a .44-46^{\circ} \mathrm{S}$ ) formations (Levi et al., 1966; Espinoza and Fuenzalida, 1971; among others) (Fig. 1). At similar latitudes on the eastern side of the Andes, coeval marine strata of the Ventana, Rio Foyel, Cerro Plataforma, La Cascada and Guadal formations have been interpreted as mainly deposited in shallower marine environments (see Encinas et al., 2018 for review).

Cordilleran-type calc-alkaline magmatism was re-established in the North Patagonian Andes by early-mid Miocene times with major pluton emplacement along the strike of the LOFZ (Pankhurst et al., 1999). This phenomenon was partly coeval with the lateral expansion of the North Patagonian Andes as evidenced by 12-18 Ma synorogenic fluvial strata in the foreland area (see Folguera et al., 2018b for review).

Intense uplift and erosion from latest Miocene times onwards are regionally recorded by apatite fission track ages (Thomson, 2002; Adriasola et al., 2005). This transpressional episode was also localised along the LOFZ and led to the exhumation of plutonic rocks of the North Patagonian Batholith.

\subsection{Local geology}

In the Aysén region, three main geological domains have been differentiated (Fig. 2): i) the latest Triassic Chonos accretionary prism in the Coast Range (Davidson et al., 1987; Hervé and Fanning, 2001; Thomson and Hervé, 2002); ii) the widespread Mesozoic-Cenozoic plutonic rocks of the North Patagonian Batholith (Pankhurst et al., 1999) together with the recent volcanic rocks of the Southern Volcanic Zone in the western slope of the main Cordillera (López-Escobar et al., 1995) and the Paleozoic Main Range Metamorphic Complex (Hervé et al., 2003); and iii) the Mesozoic to Cenozoic volcano-sedimentary successions (Haller and Lapido, 1980) overlying the Paleozoic Eastern Andes Metamorphic Complex in the eastern slope 


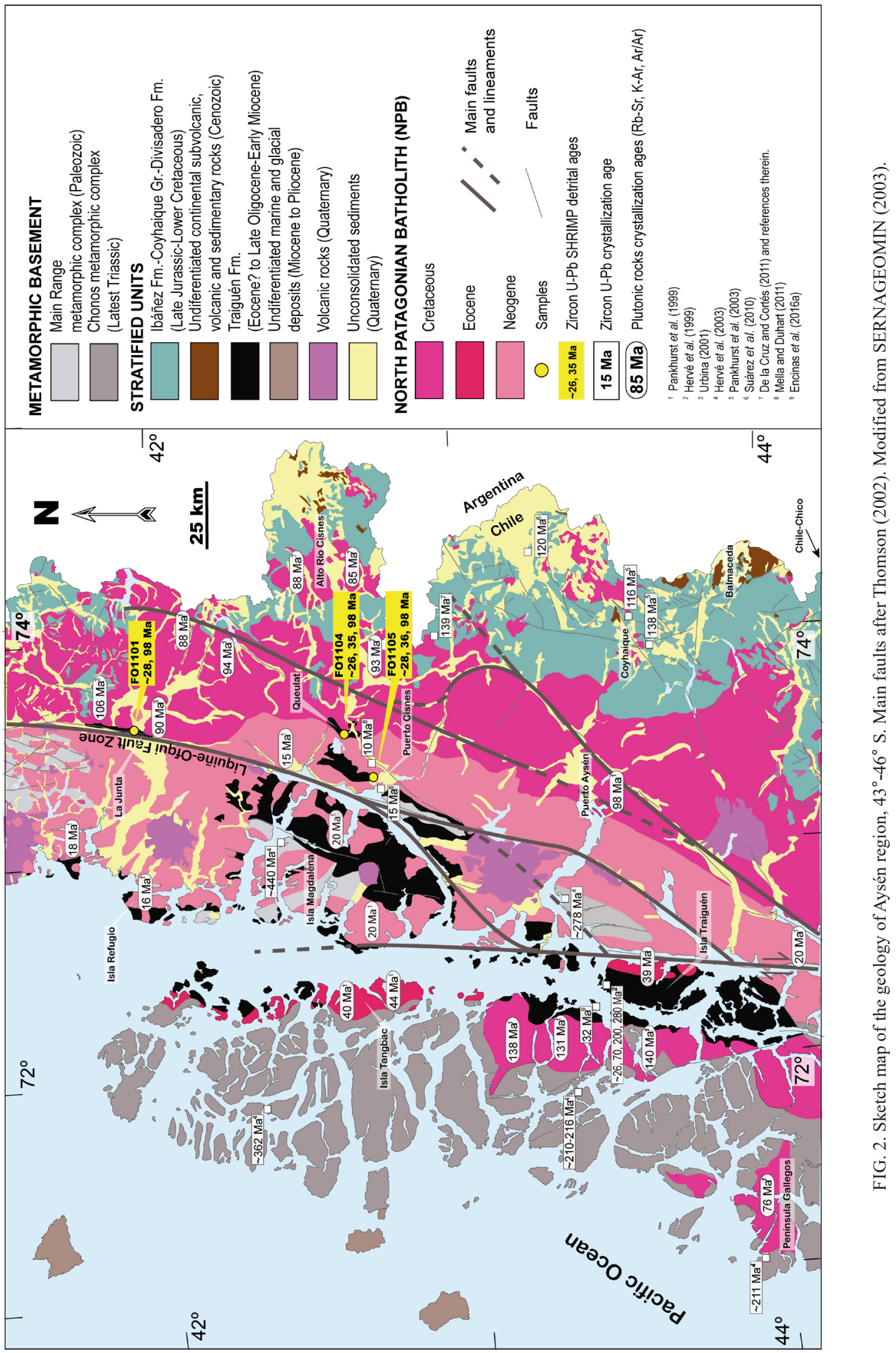


of the Andes (Hervé et al., 2003). The boundary between the first two domains is, in some areas, marked by the LOFZ (Fig. 1).

The oldest rocks recognized in the study area are the poorly known schistose, medium-to-high grade, metamorphic rocks of the Main Range Metamorphic Complex (MRMC), whose outcrops can be found in Isla Magdalena and nearby inland areas (Fig. 2). Hervé et al. (2003) reported a mid-Paleozoic maximum sedimentation age for the sedimentary protolith of a sillimanite-bearing quartz micaschist sampled in the northern shore of Isla Magdalena. No radiometric age has yet been determined for low-grade metamorphic rocks located inland at the same latitudes, in the Cuesta Queulat area and south of Puerto Cisnes, some of which have been considered to be Paleozoic (e.g., SERNAGEOMIN, 2003).

In Aysén, early-mid Paleogene volcanic and sedimentary rocks are mainly located east of the Andean Cordillera, near the Chile-Argentina international border (Fig. 2), and have been interpreted as formed in an extensional setting (e.g., Encinas et al., 2018). In particular, alkaline basalts in the Chile Chico-Balmaceda area have been related to the development of an asthenospheric window associated with Eocene subduction of the AlukFarallon seismic ridge below this portion of South America (Espinoza et al., 2005). Mid-Eocene marine strata of the Puerto Good Sequence in the Golfo de Penas area also imply extension of the forearc at that time (Forsythe et al., 1985). Apatite fission-track ages of 28-30 Ma obtained by Thomson et al. (2001) in the archipelagos north of $46^{\circ} \mathrm{S}$ indicate little denudation in the forearc since Rupelian. Restricted late Oligocene, bimodal, transitional to alkaline magmatism located in the eastern sector of Aysén has been interpreted as formed in an incipient rift setting (e.g., Morata et al., 2003).

Miocene (10-20 Ma) hornblende-biotite tonalite to granodiorite suites intrude the metamorphic rocks in the Isla Magdalena-Puerto Cisnes-Queulat area (Hervé et al., 1993; Pankhurst et al., 1999; Parada et al., 2000; Cembrano et al., 2002; Mella and Duhart, 2011). Regionally, plutonic rocks of this age are spatially associated with the LOFZ, cropping out mostly to the west of it (Pankhurst et al., 1999) (Fig. 2). Cretaceous plutonic rocks (90-120 Ma) constitute a N-S trending belt located preferentially to the east of the LOFZ (Pankhurst et al., 1992;
Pankhurst et al., 1999; Duhart, 2008), where they intrude the Late Jurassic to Early Cretaceous volcanic and sedimentary cover (Fig. 2).

\subsection{Low-grade metasedimentary rocks of the study area}

Between $43^{\circ}$ and $46^{\circ} \mathrm{S}$ the late Oligocene to early Miocene record mostly consists of marine sedimentary and volcano-sedimentary rock units. Near La Junta, sedimentary rocks of the La Junta and Vargas formations crop out along the eastern and western sides of the LOFZ, respectively (Encinas et al., 2014).

The La Junta Formation (Urbina, 2001), firstly named as "Estratos de la Junta" by Bobenrieth et al. (1983), comprises tilted successions of mainly coarse-grained sedimentary rocks (Fig. 3A) interbedded with minor andesites, cropping out in fault contact with Jurassic volcanic rocks or resting unconformably over the Lower to Upper Cretaceous pink granites of the North Patagonian Batholith. The clastic components have been interpreted as fluvial to alluvial fan deposits (Encinas et al., 2014). They include ortho-and paraconglomerates together with coarse-grained sandstones. Porphyritic andesite and rhyodacite together with coarse-grained pink granite are among the lithologies described by Urbina (2001) for the conglomerate boulders. Very low-grade metamorphic minerals (chloriteepidote-pumpellyite-prehnite) occur in vesicles or as massive granular aggregates (Urbina, 2001). Encinas et al. (2014) have reported a ca. $26 \mathrm{Ma}$ (late Oligocene) maximum sedimentation age for a $>1 \mathrm{~m}$ thick volcanic sandstone belonging to the La Junta Formation; prominent Cretaceous populations are also observed in the detrital zircon age spectra (ca. 97-108 Ma).

The marine strata of the Vargas Formation are characterized by successions of fossiliferous black shale and mudstone interbedded with finegrained sandstone and siltstone, which are found in tectonic contact with cataclastic plutonic rocks of the North Patagonian Batholith (Urbina, 2001). Conspicuous foliation led Steffen (1944) to name this unit "Formación Esquistos Arcillosos". Bobenrieth et al. (1983) included these rocks as part of "Estratos de la Junta", but they did not recognize a marine origin for them. Encinas et al. (2014) reported U-Pb LA-ICP-MS detrital zircon ages for two samples 
which yield prominent Cretaceous ( $c a .82-109 \mathrm{Ma})$ peaks, one of them also displaying a minor $(n=4)$ late Eocene (ca. $39 \mathrm{Ma}$ ) age.

Marine volcano-sedimentary rocks of the Traiguén Formation are found in the southern section of the study area, on Isla Magdalena and nearby inland areas (Espinoza and Fuenzalida, 1971; Fuenzalida and Etchart, 1975; Bobenrieth et al., 1983; Hervé et al., 1994). This formation consists of pillow basalts with mixed within-plate/volcanic-arc geochemical signatures (Hervé et al., 1995) and turbiditic sedimentary packages deposited at bathyal depths (>1,000 m) (Encinas et al., 2016a and references therein). As reported by Hervé et al. (1994), the basal contact is represented by conglomerates and breccias, with clasts of metamorphic, granitic and acid volcanic rocks, resting unconformably over the Main Range Metamorphic Complex. The same authors describe N10E-trending open folds with subvertical axial planes affecting the Traiguén Formation, with local development of axial plane cleavage in the shales. A similar strike is observed for the partly syn-sedimentary mafic dykes intruding the Traiguén Formation and metamorphic basement in the area. According to Hervé et al. (1994), the deepest part of the basin was located to the east of Isla Magdalena, next to the main trace of the LOFZ: the basin was shallower to the west, where basaltic pillow lavas are absent and acid lava flows, porphyries and breccias have been described (Hervé et al., 1994, 1995; Bobenrieth et al., 1983).

The age of deposition of this formation is constrained by a $c a$. 26 Ma maximum sedimentation age determined at its type locality on Isla Traiguén (Hervé et al., 2001), and by a U-Pb zircon age of ca. $23 \mathrm{Ma}$ for a lapilli tuff in the same area (Encinas et al., 2016a). Hervé et al. (1995) obtained two imprecise $\mathrm{Rb}$-Sr ages of $20 \pm 28 \mathrm{Ma}$ and $20 \pm 26 \mathrm{Ma}$ from sedimentary and volcanic rocks on Isla Magdalena, interpreted as related to their metamorphism. A minimum age is given by a $c a$. 20 Ma cross-cutting intrusion in the NE part of Isla Magdalena ( $\mathrm{Rb}-\mathrm{Sr}$ isochron by Pankhurst et al., 1999). A notably younger mid-Miocene ( $c a .10 \mathrm{Ma}$ ) peraluminous igneous intrusion is recognized adjacent to the LOFZ in the lower course of Río Cisnes (Mella and Duhart, 2011). It is a partly foliated leucocratic granitoid containing spessartine-rich garnet, white mica, andalusite and sillimanite as primary minerals (Hervé et al., 1993). At its eastern edge, the Río Cisnes leucogranite intrudes a sequence of pelitic hornfelses and metaconglomerates which were described by Bobenrieth et al. (1983) as metamorphosed equivalents of the Traiguén Formation.

\section{Methodology and materials}

Detrital zircon was separated from two samples of low-grade metasedimentary rocks from Puerto Cisnes-Queulat area and one paraconglomerate sample from the La Junta area by standard methods of crushing, grinding, Wilfley table, magnetic and heavy liquid separation at Universidad de Chile, Santiago. U-Pb analyses were undertaken at the Research School of Earth Sciences, The Australian National University, Canberra. The zircon grains were mounted in epoxy and polished to expose the interior. Cathodoluminescence (CL) images were obtained for all grains to select appropriate areas for analysis. U-Th-Pb analyses were carried out using sensitive high-resolution ion microprobes (SHRIMP II and SHRIMP RG) with procedures similar to those described by Williams (1998; and references therein). Sixty grains were analysed for sample FO1104 and twenty grains each for samples FO1101 and FO1105. Although 20 analyses do not give statistically complete provenance spectra, they can nevertheless help to restrict the maximum depositional age.

The analytical data were plotted on TeraWasserburg (1972) Concordia diagrams and the derived radiogenic ages were plotted on relative probability diagrams (Fig. 4) using Isoplot (Ludwig, 2003). Maximum depositional ages may be assessed by different statistical methods (see Dickinson and Gehrels, 2009): in this work we used a conservative estimate based on the mean age of the youngest cluster consisting in all cases of more than three grains. Ages for individual grains are reported at the $68 \%$ confidence level (Table 1).

Structural analysis of the studied Cenozoic units is beyond the scope of the present paper, but general structural characterization is given when considered necessary to establish some similarities with other Cenozoic units of the area.

\section{Results}

Sample FO1101 (435' $52.0^{\prime \prime}$ S/72 $\left.23^{\circ} 33.0^{\prime \prime} \mathrm{W}\right)$ was collected $1 \mathrm{~km}$ north of the town of La Junta 
and belongs to the La Junta Formation (Fig.3A). The outcrop consists of a paraconglomerate with well-rounded andesitic and minor granitic clasts $10-20 \mathrm{~cm}$ in diameter and a coarse-grained matrix with 1-2 cm clasts. The rocks do not exhibit foliation, but are affected by very low-grade regional metamorphism. The sample was taken from the matrix and the results show a prominent age peak at $98 \mathrm{Ma}$ but with five of the twenty zircon grains giving ages of 25-31 Ma: three of these yield a consistent weighted mean age of $28 \pm 1$ Ma (Fig. 4).

Sample FO1104 ( $\left.44^{\circ} 38^{\prime} 21.3^{\prime \prime} \mathrm{S} / 72^{\circ} 26^{\prime} 23.4^{\prime \prime} \mathrm{W}\right)$ is a foliated metaconglomerate from Queulat area with flattened clasts 1 to $5 \mathrm{~cm}$ long immersed in a greenish matrix (Fig. 3C). The metaconglomerate occurs within a thick pile of alternating packages of green phyllite and metasandstone, resembling turbidite deposits (Fig 3B, D). The So is NNE/40WNW. They contain isoclinally folded calcite (Fig. 3E) veins together with a $\mathrm{N} 80 \mathrm{~W} / 20 \mathrm{~N}$ main axial plane foliation (Sp). Structures developed on the foliation plane indicate dextral shear (Fig 3F). Nearly vertical, green microdiorite post-tectonic dykes a few metres wide, some with slightly sinuous margins, cross-cut the low-grade metamorphic rocks (Fig. 3B). The more complete detrital zircon spectrum for this sample exhibits a prominent age peak at $35 \mathrm{Ma}(\mathrm{n}=10)$, clearly indicating a midCenozoic maximum depositional age. However, ages tail down to a minimum of $c a .23 \mathrm{Ma}$, with two possible minor age peaks at $26 \mathrm{Ma}(\mathrm{n}=3)$ and $29 \mathrm{Ma}(\mathrm{n}=4)$. In addition, there are older age peaks at 39 and $96 \mathrm{Ma}$ (Fig. 4).

Sample FO1105 (44²5’33.3” S/72 $\left.38^{\prime} 50.9^{\prime \prime} \mathrm{W}\right)$ is a schist from a cutting in the Puerto CisnesQueulat road. It is well foliated and banded, with post-tectonic randomly-orientated biotite microporphyroblasts in a quartz-rich matrix. The Sp $\mathrm{N} 50 \mathrm{E} / 58 \mathrm{NW}$ fabric is a crenulation cleavage. The detrital zircon ages (Fig. 4) show evidence for both Permian (ca. $290 \mathrm{Ma}$ ) and Cretaceous (ca. $130 \mathrm{Ma}$ ) provenance, but 16 of the 20 grains gave Paleogene ages of 26-39 Ma, half of these being Oligocene. A conservative estimate of the maximum sedimentation age is $28 \pm 1 \mathrm{Ma}$, given by the youngest 6 ages, albeit with some scatter beyond the analytical uncertainty. The well-defined peak at $c a .39 \mathrm{Ma}(\mathrm{n}=6)$ also appears to represent a significant provenance age (Fig. 4).

\section{Discussion}

\subsection{Age of the sedimentary protolith and stratigraphic correlations with geological units of the North Patagonian Andes}

The $c a .28 \mathrm{Ma}$ age obtained here for detrital zircon in the two metamorphic rocks from the Puerto Cisnes-Queulat area (Fig. 4), implies probable late Oligocene (Rupelian-Chattian) deposition. It precludes previous correlation with the Paleozoic Main Range Metamorphic Complex (e.g., SERNAGEOMIN, 2003), but validates the correlation of these rocks with the Traiguén Formation (Hervé et al., 2001; Encinas et al., 2016a), as previously suggested by Bobenrieth et al. (1983). Therefore, these rocks constitute the easternmost known outcrops of the Traiguén Formation.

In the North Patagonian Andes of Chile at $42^{\circ}-46^{\circ} \mathrm{S}$, most of the late Oligocene-early Miocene marine strata (Ayacara, Puduhuapi, Vargas and Traiguén formations) are located to the west of a N-S trending belt of (mostly) Lower to Upper Cretaceous plutonic rocks belonging to the North Patagonian Batholith (Pankhurst et al., 1992; Pankhurst et al., 1999; Duhart, 2008) (Fig. 1). These volcanosedimentary units share sedimentological features indicative of outer-shelf to deep-sea deposition in basins located adjacent to coeval volcanic edifices (e.g., Levi et al., 1966; Encinas et al., 2013). On the other hand, restricted outcrops of late Oligocene-early Miocene strata deposited in a shallow-marine environment are located in the eastern reaches of the batholith (e.g., La Cascada Formation, Thiele et al., 1978) or tens of $\mathrm{km}$ farther east (e.g., Guadal Formation). Based on paleontological constraints, an Atlantic affinity is well-documented for the Guadal Formation (Frassinetti and Covacevich, 1999; among others), contrasting with the Pacific affinity of the marine units to the west. The affinity of sedimentary rocks of the La Cascada Formation is more debatable (Encinas et al., 2014), as its position east of the Cretaceous batholith and shallow sedimentary environment show marked differences with the above mentioned marine formations located near to or west of the LOFZ.

The only known non-marine strata in the western Andean slope are the coarse-grained (alluvial?) 


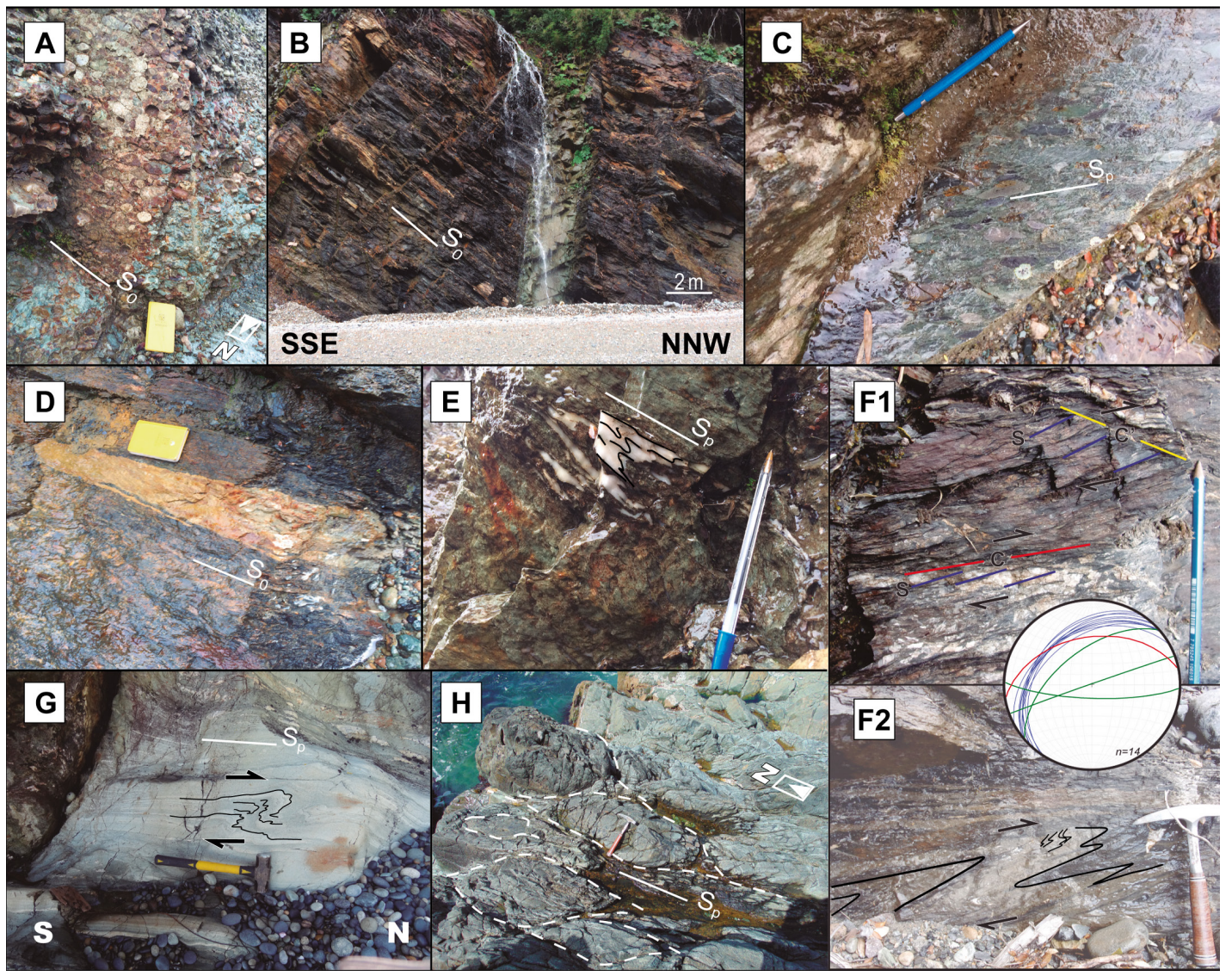

FIG. 3. Outcrop images of A. La Junta Formation at its type locality; B-F. Traiguén Formation in the Queulat area; G-H. Traiguén Formation on Isla Refugio. B. Tilted and foliated packages of shales and minor sandstones intruded by a post-tectonic microdiorite dyke; C. dated metaconglomerate with flattened volcanic clasts; D. yellowish sandstone interpreted as a synsedimetary dyke crosscutting foliated shales with thin sandstone intercalations; E. isoclinally folded calcite veins; (F1) S-C structures developed in the low grade metasediments-C' structures, in yellow, are also recognized (F2) discrete dextral shear zone in the metasediments. Stereonet data, blue: S structures, red: C structures, green: post-tectonic dyke orientation. G. Foliated metavolcanic rocks with a dextral sense of shear; H. partly preserved massive pillow lavas with foliated inter-pillow material. $\mathbf{S}_{\mathbf{0}}$ : bedding. $\mathbf{S}_{\mathbf{p}}$ : main foliation.

sedimentary rocks of the La Junta Formation. According to Bobenrieth et al. (1983), in the Queulat area there is a package of coarse-grained conglomerate with $5-10 \mathrm{~cm}$ clasts of dacitic, andesitic and granitic composition disposed in a tuffaceous matrix intensely deformed by dynamic metamorphism. These authors correlated this with the La Junta Formation, whose type locality is $70 \mathrm{~km}$ further north. When the detrital zircon age spectra of the Queulat metaconglomerate (sample FO1104) is compared with that of the La Junta coarse-grained paraconglomerate (sample FO1101), similar maximum possible sedimentation ages are obtained as well as the same major Cenomanian age provenance (96-99 Ma). These results are in agreement with those obtained by Encinas et al. (2014) for the same unit (sample JUN-1, Table 2), suggesting that they are coetaneous and that the La Junta Formation may be a high-energy lateral facies variant of the Traiguén Formation.

At Caleta Ayacara (Fig. 1), the base of the exposed lower member of the Ayacara Formation (Levi et al., 1966) consists of mostly massive (ortho-and para-) conglomerates, tens of metres thick with subangular to rounded clasts whose size varies from millimetres to several tens of centimetres. Aphanitic and trachytic andesite, tuff and minor dacite are the most common lithology for the clasts, as well as plutonic rocks here (Encinas et al., 2013). These 

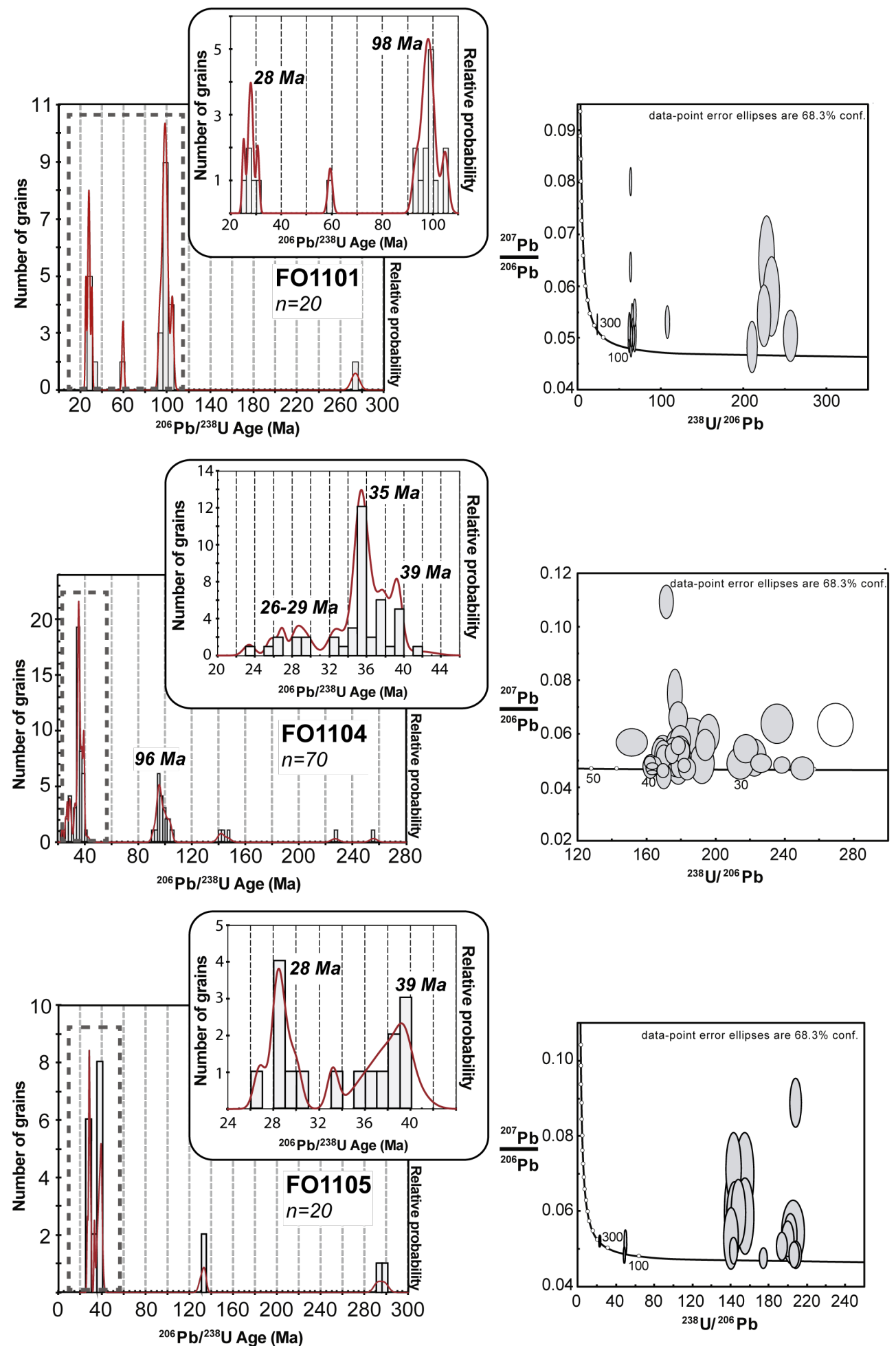

FIG. 4. U-Pb plots for zircon data from the three samples analysed in this study. Tera-Wasserburg plots (right-hand side) and age versus probability plots (left-hand side). 
TABLE 1. SUMMARY OF U-Pb SHRIMP RESULTS FOR ANALYZED ZIRCONS.

\begin{tabular}{|c|c|c|c|c|c|c|c|c|c|c|c|c|c|c|}
\hline \multirow{2}{*}{$\begin{array}{c}\text { Grain } \\
\text { spot }\end{array}$} & \multirow{2}{*}{$\underset{(\mathbf{p p m})}{\mathrm{U}}$} & \multirow{2}{*}{$\begin{array}{c}\text { Th } \\
\text { (ppm) }\end{array}$} & \multirow{2}{*}{$\mathbf{T h} / \mathbf{U}$} & \multirow{2}{*}{$\begin{array}{l}{ }^{206} \mathrm{~Pb}^{*} \\
(\mathrm{ppm})\end{array}$} & \multirow{2}{*}{ 年44 $\mathrm{Pb} /$} & \multirow{2}{*}{$\begin{array}{l}\mathbf{f}_{206} \\
\%\end{array}$} & \multicolumn{4}{|c|}{ Total } & \multicolumn{2}{|c|}{ Radiogenic } & \multicolumn{2}{|c|}{ Age (Ma) } \\
\hline & & & & & & & $\begin{array}{l}{ }^{238} \mathrm{U} / \\
{ }^{206} \mathrm{~Pb}\end{array}$ & \pm & $\begin{array}{l}{ }^{207} \mathrm{~Pb} / \\
{ }^{206} \mathrm{~Pb}\end{array}$ & \pm & $\begin{array}{c}{ }^{206} \mathrm{~Pb} / \\
{ }^{238} \mathbf{U}\end{array}$ & \pm & $\begin{array}{c}{ }^{206} \mathbf{P b} / \\
{ }^{238} \mathbf{U}\end{array}$ & \pm \\
\hline \multicolumn{15}{|c|}{ Sample FO1101 - La Junta paraconglomerate } \\
\hline 1.1 & 224 & 151 & 0.68 & 0.8 & 0.000584 & 0.48 & 255.72 & 5.70 & 0.0503 & 0.0033 & 0.0039 & 0.0001 & 25.0 & 0.6 \\
\hline 2.1 & 238 & 138 & 0.58 & 3.1 & 0.000275 & 0.30 & 65.04 & 0.94 & 0.0504 & 0.0021 & 0.0153 & 0.0002 & 98.1 & 1.4 \\
\hline 3.1 & 1,239 & 1,191 & 0.96 & 17.5 & 0.000167 & 0.07 & 60.89 & 0.67 & 0.0487 & 0.0007 & 0.0164 & 0.0002 & 104.9 & 1.1 \\
\hline 4.1 & 207 & 308 & 1.49 & 2.6 & 0.001325 & 0.86 & 68.11 & 0.97 & 0.0547 & 0.0018 & 0.0146 & 0.0002 & 93.2 & 1.3 \\
\hline 5.1 & 183 & 159 & 0.87 & 0.7 & - & 0.19 & 209.88 & 4.14 & 0.0481 & 0.0033 & 0.0048 & 0.0001 & 30.6 & 0.6 \\
\hline 6.1 & 130 & 76 & 0.58 & 0.5 & 0.003252 & 0.96 & 223.90 & 5.23 & 0.0542 & 0.0040 & 0.0044 & 0.0001 & 28.5 & 0.7 \\
\hline 7.1 & 89 & 55 & 0.62 & 0.3 & 0.003331 & 2.35 & 226.99 & 6.15 & 0.0651 & 0.0055 & 0.0043 & 0.0001 & 27.7 & 0.8 \\
\hline 8.1 & 304 & 161 & 0.53 & 4.1 & 0.000326 & 0.27 & 63.95 & 0.83 & 0.0501 & 0.0014 & 0.0156 & 0.0002 & 99.8 & 1.3 \\
\hline 9.1 & 90 & 48 & 0.54 & 0.3 & 0.004517 & 1.43 & 233.10 & 6.52 & 0.0579 & 0.0051 & 0.0042 & 0.0001 & 27.2 & 0.8 \\
\hline 10.1 & 123 & 70 & 0.57 & 4.6 & 0.000634 & 0.10 & 23.02 & 0.32 & 0.0526 & 0.0014 & 0.0434 & 0.0006 & 273.8 & 3.8 \\
\hline 11.1 & 68 & 49 & 0.73 & 0.9 & 0.000592 & 0.30 & 63.67 & 1.24 & 0.0504 & 0.0028 & 0.0157 & 0.0003 & 100.2 & 2.0 \\
\hline 12.1 & 143 & 176 & 1.23 & 1.9 & 0.001644 & 0.70 & 64.64 & 1.00 & 0.0535 & 0.0020 & 0.0154 & 0.0002 & 98.3 & 1.5 \\
\hline 13.1 & 231 & 134 & 0.58 & 1.8 & 0.000770 & 0.73 & 107.41 & 1.65 & 0.0530 & 0.0021 & 0.0092 & 0.0001 & 59.3 & 0.9 \\
\hline 14.1 & 103 & 71 & 0.69 & 1.3 & 0.000682 & 0.36 & 67.86 & 1.18 & 0.0508 & 0.0024 & 0.0147 & 0.0003 & 94.0 & 1.7 \\
\hline 15.1 & 313 & 429 & 1.37 & 4.0 & 0.001812 & 0.29 & 66.49 & 0.87 & 0.0502 & 0.0014 & 0.0150 & 0.0002 & 96.0 & 1.3 \\
\hline 16.1 & 177 & 202 & 1.14 & 2.3 & 0.000222 & 0.38 & 66.01 & 0.97 & 0.0510 & 0.0018 & 0.0151 & 0.0002 & 96.6 & 1.4 \\
\hline 17.1 & 120 & 124 & 1.03 & 1.7 & - & 0.35 & 60.99 & 0.97 & 0.0509 & 0.0025 & 0.0163 & 0.0003 & 104.5 & 1.7 \\
\hline 18.1 & 273 & 259 & 0.95 & 3.7 & 0.002428 & 4.07 & 62.93 & 0.83 & 0.0803 & 0.0018 & 0.0152 & 0.0002 & 97.5 & 1.3 \\
\hline 19.1 & 147 & 138 & 0.94 & 1.9 & 0.000592 & 0.49 & 64.81 & 1.02 & 0.0519 & 0.0020 & 0.0154 & 0.0002 & 98.2 & 1.6 \\
\hline 20.1 & 192 & 266 & 1.38 & 2.6 & 0.001668 & 1.97 & 62.72 & 0.90 & 0.0637 & 0.0019 & 0.0156 & 0.0002 & 100.0 & 1.5 \\
\hline \multicolumn{15}{|c|}{ Sample FO1104 - Queulat metaconglomerate } \\
\hline 1.1 & 354 & 421 & 1.19 & 1.3 & 0.001520 & 0.40 & 226.26 & 4.00 & 0.0498 & 0.0023 & 0.0044 & 0.0001 & 28.3 & 0.5 \\
\hline 2.1 & 110 & 95 & 0.87 & 0.5 & 0.002000 & 0.67 & 178.17 & 4.58 & 0.0521 & 0.0041 & 0.0056 & 0.0001 & 35.8 & 0.9 \\
\hline 3.1 & 50 & 37 & 0.75 & 0.2 & 0.014692 & 1.31 & 186.10 & 6.92 & 0.0571 & 0.0061 & 0.0053 & 0.0002 & 34.1 & 1.3 \\
\hline 4.1 & 317 & 336 & 1.06 & 4.1 & 0.000170 & 0.04 & 66.42 & 0.91 & 0.0482 & 0.0013 & 0.0151 & 0.0002 & 96.3 & 1.3 \\
\hline 5.1 & 125 & 76 & 0.61 & 0.6 & 0.002612 & 1.46 & 179.67 & 3.82 & 0.0582 & 0.0036 & 0.0055 & 0.0001 & 35.3 & 0.8 \\
\hline 6.1 & 82 & 85 & 1.04 & 1.1 & 0.000000 & 0.26 & 66.11 & 1.22 & 0.0500 & 0.0026 & 0.0151 & 0.0003 & 96.5 & 1.8 \\
\hline 7.1 & 144 & 131 & 0.91 & 2.0 & 0.000130 & 0.08 & 61.88 & 0.93 & 0.0487 & 0.0018 & 0.0161 & 0.0002 & 103.3 & 1.6 \\
\hline 8.1 & 154 & 100 & 0.65 & 0.6 & 0.003203 & 1.06 & 217.44 & 4.59 & 0.0550 & 0.0035 & 0.0046 & 0.0001 & 29.3 & 0.6 \\
\hline 9.1 & 160 & 90 & 0.56 & 0.8 & - & 1.02 & 179.46 & 3.58 & 0.0548 & 0.0034 & 0.0055 & 0.0001 & 35.5 & 0.7 \\
\hline 10.1 & 227 & 94 & 0.41 & 2.9 & 0.000596 & 0.03 & 66.91 & 0.92 & 0.0482 & 0.0015 & 0.0149 & 0.0002 & 95.6 & 1.3 \\
\hline 11.1 & 140 & 130 & 0.93 & 0.7 & 0.001042 & 0.92 & 175.93 & 3.58 & 0.0540 & 0.0034 & 0.0056 & 0.0001 & 36.2 & 0.8 \\
\hline 12.1 & 761 & 491 & 0.65 & 10.2 & 0.000071 & 0.04 & 64.30 & 0.72 & 0.0483 & 0.0008 & 0.0155 & 0.0002 & 99.4 & 1.1 \\
\hline 13.1 & 126 & 69 & 0.55 & 0.5 & 0.005636 & 0.40 & 214.40 & 4.99 & 0.0498 & 0.0038 & 0.0046 & 0.0001 & 29.9 & 0.7 \\
\hline 14.1 & 266 & 103 & 0.39 & 0.9 & - & 0.17 & 250.26 & 4.59 & 0.0479 & 0.0028 & 0.0040 & 0.0001 & 25.7 & 0.5 \\
\hline 15.1 & 117 & 70 & 0.60 & 0.4 & 0.003218 & 2.24 & 235.50 & 5.97 & 0.0643 & 0.0048 & 0.0042 & 0.0001 & 26.7 & 0.7 \\
\hline 16.1 & 171 & 107 & 0.63 & 2.4 & 0.000373 & 0.33 & 61.95 & 0.90 & 0.0507 & 0.0017 & 0.0161 & 0.0002 & 102.9 & 1.5 \\
\hline 17.1 & 181 & 110 & 0.61 & 5.6 & 0.000195 & $<0.01$ & 27.93 & 0.35 & 0.0497 & 0.0011 & 0.0359 & 0.0005 & 227.1 & 2.8 \\
\hline 18.1 & 91 & 77 & 0.84 & 1.7 & 0.000906 & 0.35 & 45.09 & 0.74 & 0.0517 & 0.0024 & 0.0221 & 0.0004 & 140.9 & 2.3 \\
\hline 19.1 & 187 & 96 & 0.51 & 2.4 & - & 0.06 & 67.18 & 0.99 & 0.0484 & 0.0018 & 0.0149 & 0.0002 & 95.2 & 1.4 \\
\hline 20.1 & 440 & 292 & 0.66 & 5.7 & 0.000482 & $<0.01$ & 65.94 & 0.80 & 0.0475 & 0.0011 & 0.0152 & 0.0002 & 97.1 & 1.2 \\
\hline 21.1 & 582 & 285 & 0.49 & 2.7 & 0.000256 & 0.28 & 181.83 & 2.48 & 0.0490 & 0.0018 & 0.0055 & 0.0001 & 35.3 & 0.5 \\
\hline 22.1 & 133 & 114 & 0.85 & 0.7 & 0.003237 & 1.19 & 177.05 & 3.77 & 0.0562 & 0.0039 & 0.0056 & 0.0001 & 35.9 & 0.8 \\
\hline 23.1 & 92 & 75 & 0.82 & 1.8 & 0.000706 & 0.69 & 44.56 & 0.68 & 0.0544 & 0.0023 & 0.0223 & 0.0003 & 142.1 & 2.2 \\
\hline 24.1 & 146 & 114 & 0.78 & 1.8 & 0.001008 & 1.10 & 69.47 & 1.09 & 0.0566 & 0.0024 & 0.0142 & 0.0002 & 91.1 & 1.4 \\
\hline
\end{tabular}


Table 1 continued.

\begin{tabular}{|c|c|c|c|c|c|c|c|c|c|c|c|c|c|c|}
\hline \multirow{2}{*}{$\begin{array}{l}\text { Grain } \\
\text { spot }\end{array}$} & \multirow{2}{*}{$\begin{array}{c}\mathbf{U} \\
(\mathbf{p p m})\end{array}$} & \multirow{2}{*}{$\begin{array}{c}\text { Th } \\
(\mathrm{ppm})\end{array}$} & \multirow{2}{*}{$\mathbf{T h} / \mathbf{U}$} & \multirow{2}{*}{$\begin{array}{l}{ }^{206} \mathrm{~Pb}^{*} \\
\text { (ppm) }\end{array}$} & \multirow{2}{*}{$\begin{array}{l}{ }^{204} \mathrm{~Pb} / \\
{ }^{206} \mathrm{~Pb}\end{array}$} & \multirow{2}{*}{$\begin{array}{c}f_{206} \\
\%\end{array}$} & \multicolumn{4}{|c|}{ Total } & \multicolumn{2}{|c|}{ Radiogenic } & \multicolumn{2}{|c|}{ Age (Ma) } \\
\hline & & & & & & & $\begin{array}{l}{ }^{238} \mathrm{U} / \\
{ }^{206} \mathrm{~Pb}\end{array}$ & \pm & $\begin{array}{l}{ }^{207} \mathrm{~Pb} / \\
{ }^{206} \mathrm{~Pb}\end{array}$ & \pm & $\begin{array}{c}{ }^{206} \mathrm{~Pb} / \\
{ }^{238} \mathrm{U}\end{array}$ & \pm & $\begin{array}{l}{ }^{206} \mathrm{~Pb} / \\
{ }^{238} \mathrm{U}\end{array}$ & \pm \\
\hline 26.1 & 183 & 115 & 0.62 & 0.9 & 0.000899 & $<0.01$ & 170.23 & 2.97 & 0.0449 & 0.0032 & 0.0059 & 0.0001 & 37.8 & 0.7 \\
\hline 27.1 & 149 & 71 & 0.47 & 2.0 & 0.001748 & 0.33 & 64.72 & 0.98 & 0.0506 & 0.0022 & 0.0154 & 0.0002 & 98.5 & 1.5 \\
\hline 28.1 & 119 & 57 & 0.48 & 0.5 & 0.001131 & 1.76 & 196.00 & 4.16 & 0.0606 & 0.0047 & 0.0050 & 0.0001 & 32.2 & 0.7 \\
\hline 29.1 & 8,966 & 7,393 & 0.82 & 47.1 & 0.000015 & $<0.01$ & 163.58 & 1.84 & 0.0463 & 0.0006 & 0.0061 & 0.0001 & 39.3 & 0.4 \\
\hline 30.1 & 285 & 218 & 0.77 & 1.4 & 0.000288 & 0.44 & 180.85 & 2.94 & 0.0502 & 0.0031 & 0.0055 & 0.0001 & 35.4 & 0.6 \\
\hline 31.1 & 608 & 588 & 0.97 & 3.2 & 0.000141 & 0.16 & 163.25 & 2.09 & 0.0481 & 0.0017 & 0.0061 & 0.0001 & 39.3 & 0.5 \\
\hline 32.1 & 657 & 874 & 1.33 & 3.5 & 0.000481 & 0.41 & 162.40 & 2.12 & 0.0501 & 0.0016 & 0.0061 & 0.0001 & 39.4 & 0.5 \\
\hline 33.1 & 107 & 147 & 1.38 & 1.5 & 0.000652 & 0.69 & 62.70 & 0.98 & 0.0535 & 0.0025 & 0.0158 & 0.0003 & 101.3 & 1.6 \\
\hline 34.1 & 238 & 177 & 0.74 & 1.2 & 0.005122 & 7.91 & 171.62 & 2.80 & 0.1093 & 0.0041 & 0.0054 & 0.0001 & 34.5 & 0.6 \\
\hline 34.2 & 83 & 59 & 0.71 & 0.4 & - & 0.77 & 169.87 & 3.94 & 0.0529 & 0.0047 & 0.0058 & 0.0001 & 37.5 & 0.9 \\
\hline 35.1 & 2,836 & 1,348 & 0.48 & 14.9 & - & 0.24 & 163.41 & 2.16 & 0.0487 & 0.0008 & 0.0061 & 0.0001 & 39.2 & 0.5 \\
\hline 36.1 & 630 & 378 & 0.60 & 2.3 & 0.000431 & 0.31 & 238.27 & 3.21 & 0.0490 & 0.0019 & 0.0042 & 0.0001 & 26.9 & 0.4 \\
\hline 37.1 & 137 & 87 & 0.64 & 1.8 & - & $<0.01$ & 64.07 & 1.23 & 0.0452 & 0.0021 & 0.0157 & 0.0003 & 100.2 & 1.9 \\
\hline 38.1 & 176 & 169 & 0.96 & 3.6 & 0.001411 & 2.02 & 42.53 & 0.71 & 0.0651 & 0.0019 & 0.0230 & 0.0004 & 146.8 & 2.5 \\
\hline 39.1 & 398 & 289 & 0.73 & 1.9 & 0.000922 & 1.21 & 178.53 & 2.60 & 0.0563 & 0.0022 & 0.0055 & 0.0001 & 35.6 & 0.5 \\
\hline 40.1 & 143 & 116 & 0.81 & 0.5 & - & 2.20 & 269.31 & 6.78 & 0.0639 & 0.0052 & 0.0036 & 0.0001 & 23.4 & 0.6 \\
\hline 41.1 & 213 & 138 & 0.65 & 1.0 & 0.001339 & 0.63 & 179.28 & 2.98 & 0.0517 & 0.0030 & 0.0055 & 0.0001 & 35.6 & 0.6 \\
\hline 42.1 & 198 & 108 & 0.54 & 0.9 & 0.000000 & 0.63 & 181.25 & 3.35 & 0.0517 & 0.0031 & 0.0055 & 0.0001 & 35.2 & 0.7 \\
\hline 43.1 & 57 & 56 & 0.98 & 1.2 & 0.023904 & 39.64 & 41.73 & 1.60 & 0.3627 & 0.0520 & 0.0145 & 0.0017 & 92.6 & 10.7 \\
\hline 44.1 & 216 & 111 & 0.51 & 1.0 & 0.001941 & 3.64 & 176.68 & 2.93 & 0.0755 & 0.0058 & 0.0055 & 0.0001 & 35.1 & 0.6 \\
\hline 45.1 & 194 & 145 & 0.75 & 0.9 & 0.000026 & 2.50 & 178.67 & 3.44 & 0.0665 & 0.0038 & 0.0055 & 0.0001 & 35.1 & 0.7 \\
\hline 46.1 & 77 & 44 & 0.58 & 1.0 & 0.000873 & 0.44 & 67.41 & 1.34 & 0.0514 & 0.0031 & 0.0148 & 0.0003 & 94.5 & 1.9 \\
\hline 47.1 & 159 & 196 & 1.23 & 0.7 & 0.001238 & 1.23 & 194.18 & 3.67 & 0.0564 & 0.0038 & 0.0051 & 0.0001 & 32.7 & 0.6 \\
\hline 48.1 & 556 & 442 & 0.79 & 2.8 & - & 0.03 & 169.89 & 2.31 & 0.0470 & 0.0017 & 0.0059 & 0.0001 & 37.8 & 0.5 \\
\hline 49.1 & 160 & 112 & 0.70 & 0.8 & 0.001341 & 0.76 & 169.63 & 3.09 & 0.0528 & 0.0035 & 0.0059 & 0.0001 & 37.6 & 0.7 \\
\hline 50.1 & 801 & 285 & 0.36 & 27.9 & 0.000112 & 0.09 & 24.68 & 0.27 & 0.0520 & 0.0006 & 0.0405 & 0.0004 & 255.8 & 2.7 \\
\hline 51.1 & 76 & 51 & 0.67 & 0.3 & 0.004744 & 0.36 & 192.20 & 4.73 & 0.0496 & 0.0049 & 0.0052 & 0.0001 & 33.3 & 0.8 \\
\hline 52.1 & 188 & 157 & 0.83 & 2.5 & 0.000319 & 0.36 & 65.83 & 0.89 & 0.0508 & 0.0019 & 0.0151 & 0.0002 & 96.8 & 1.3 \\
\hline 53.1 & 279 & 215 & 0.77 & 1.4 & 0.000573 & 0.83 & 175.06 & 2.64 & 0.0533 & 0.0025 & 0.0057 & 0.0001 & 36.4 & 0.6 \\
\hline 54.1 & 76 & 53 & 0.70 & 1.0 & 0.000557 & 0.59 & 63.61 & 1.11 & 0.0527 & 0.0030 & 0.0156 & 0.0003 & 100.0 & 1.8 \\
\hline 55.1 & 88 & 55 & 0.62 & 0.4 & 0.001960 & 0.25 & 178.63 & 4.11 & 0.0487 & 0.0045 & 0.0056 & 0.0001 & 35.9 & 0.8 \\
\hline 56.1 & 278 & 299 & 1.08 & 1.3 & - & 0.11 & 183.80 & 3.08 & 0.0476 & 0.0027 & 0.0054 & 0.0001 & 34.9 & 0.6 \\
\hline 57.1 & 159 & 124 & 0.78 & 2.0 & 0.000650 & 0.26 & 67.90 & 0.99 & 0.0500 & 0.0021 & 0.0147 & 0.0002 & 94.0 & 1.4 \\
\hline 58.1 & 91 & 85 & 0.94 & 1.1 & 0.000371 & 0.14 & 68.74 & 1.17 & 0.0490 & 0.0028 & 0.0145 & 0.0003 & 93.0 & 1.6 \\
\hline 59.1 & 240 & 162 & 0.68 & 1.2 & 0.001480 & 0.24 & 178.17 & 4.76 & 0.0486 & 0.0027 & 0.0056 & 0.0002 & 36.0 & 1.0 \\
\hline 60.1 & 201 & 182 & 0.91 & 1.0 & 0.001051 & 0.27 & 179.09 & 3.00 & 0.0489 & 0.0029 & 0.0056 & 0.0001 & 35.8 & 0.6 \\
\hline 61.1 & 198 & 140 & 0.71 & 1.0 & 0.000757 & 0.66 & 169.94 & 2.80 & 0.0520 & 0.0029 & 0.0058 & 0.0001 & 37.6 & 0.6 \\
\hline 62.1 & 221 & 154 & 0.70 & 3.1 & 0.000564 & 0.94 & 60.58 & 0.81 & 0.0556 & 0.0019 & 0.0164 & 0.0002 & 104.6 & 1.4 \\
\hline 63.1 & 381 & 181 & 0.47 & 4.9 & 0.000405 & 0.27 & 66.98 & 0.84 & 0.0500 & 0.0014 & 0.0149 & 0.0002 & 95.3 & 1.2 \\
\hline 64.1 & 256 & 193 & 0.75 & 1.2 & 0.001009 & 0.92 & 180.69 & 2.86 & 0.0540 & 0.0028 & 0.0055 & 0.0001 & 35.3 & 0.6 \\
\hline 65.1 & 199 & 97 & 0.49 & 1.1 & 0.000688 & 0.21 & 162.58 & 2.77 & 0.0485 & 0.0029 & 0.0061 & 0.0001 & 39.4 & 0.7 \\
\hline 66.1 & 359 & 355 & 0.99 & 1.9 & 0.000452 & 0.24 & 165.09 & 2.35 & 0.0487 & 0.0021 & 0.0060 & 0.0001 & 38.8 & 0.6 \\
\hline 67.1 & 268 & 191 & 0.71 & 3.4 & 0.000252 & 0.39 & 67.32 & 0.88 & 0.0510 & 0.0017 & 0.0148 & 0.0002 & 94.7 & 1.3 \\
\hline 68.1 & 134 & 92 & 0.69 & 0.7 & 0.000571 & 0.82 & 168.17 & 3.23 & 0.0533 & 0.0037 & 0.0059 & 0.0001 & 37.9 & 0.7 \\
\hline 69.1 & 173 & 136 & 0.79 & 0.7 & 0.000244 & 0.65 & 222.61 & 4.56 & 0.0517 & 0.0046 & 0.0045 & 0.0001 & 28.7 & 0.6 \\
\hline 70.1 & 157 & 69 & 0.44 & 0.9 & 0.001312 & 1.34 & 151.93 & 5.90 & 0.0575 & 0.0033 & 0.0065 & 0.0003 & 41.7 & 1.6 \\
\hline
\end{tabular}


Table 1 continued.

\begin{tabular}{|c|c|c|c|c|c|c|c|c|c|c|c|c|c|c|}
\hline \multirow{2}{*}{$\begin{array}{c}\text { Grain } \\
\text { spot }\end{array}$} & \multirow{2}{*}{$\begin{array}{c}\mathrm{U} \\
(\mathbf{p p m})\end{array}$} & \multirow{2}{*}{$\begin{array}{c}\text { Th } \\
\text { (ppm) }\end{array}$} & \multirow{2}{*}{$\mathbf{T h} / \mathbf{U}$} & \multirow{2}{*}{$\begin{array}{l}{ }^{206} \mathrm{~Pb}^{*} \\
(\mathrm{ppm})\end{array}$} & \multirow{2}{*}{$\begin{array}{l}{ }^{204} \mathrm{~Pb} / \\
{ }^{206} \mathrm{~Pb}\end{array}$} & \multirow{2}{*}{$\begin{array}{l}\mathbf{f}_{206} \\
\%\end{array}$} & \multicolumn{4}{|c|}{ Total } & \multicolumn{2}{|c|}{ Radiogenic } & \multicolumn{2}{|c|}{ Age (Ma) } \\
\hline & & & & & & & $\begin{array}{l}{ }^{238} \mathbf{U} / \\
{ }^{206} \mathbf{P b}\end{array}$ & \pm & $\begin{array}{l}{ }^{207} \mathrm{~Pb} / \\
{ }^{206} \mathrm{~Pb}\end{array}$ & \pm & $\begin{array}{c}{ }^{206} \mathbf{P b} / \\
{ }^{238} \mathbf{U}\end{array}$ & \pm & $\begin{array}{c}{ }^{206} \mathrm{~Pb} / \\
{ }^{238} \mathbf{U}\end{array}$ & \pm \\
\hline \multicolumn{15}{|c|}{ Sample FO1105 - Puerto Cisnes schist } \\
\hline 1.1 & 50 & 40 & 0.80 & 0.2 & 0.001036 & 2.95 & 174.12 & 6.27 & 0.0701 & 0.0072 & 0.0056 & 0.0002 & 35.8 & 1.3 \\
\hline 2.1 & 68 & 30 & 0.44 & 0.3 & 0.005658 & 1.03 & 224.53 & 7.77 & 0.0547 & 0.0056 & 0.0044 & 0.0002 & 28.4 & 1.0 \\
\hline 3.1 & 364 & 126 & 0.35 & 1.4 & 0.001618 & 0.28 & 227.05 & 3.74 & 0.0488 & 0.0023 & 0.0044 & 0.0001 & 28.3 & 0.5 \\
\hline 4.1 & 41 & 35 & 0.85 & 0.2 & 0.004602 & 1.50 & 163.28 & 5.40 & 0.0587 & 0.0062 & 0.0060 & 0.0002 & 38.8 & 1.3 \\
\hline 5.1 & 254 & 102 & 0.40 & 1.3 & 0.001764 & 0.38 & 162.27 & 2.63 & 0.0498 & 0.0023 & 0.0061 & 0.0001 & 39.5 & 0.6 \\
\hline 6.1 & 209 & 108 & 0.52 & 8.3 & 0.000171 & 0.03 & 21.59 & 0.26 & 0.0524 & 0.0009 & 0.0463 & 0.0006 & 291.8 & 3.5 \\
\hline 7.1 & 33 & 23 & 0.70 & 0.2 & 0.003917 & 1.65 & 161.64 & 6.04 & 0.0599 & 0.0080 & 0.0061 & 0.0002 & 39.1 & 1.5 \\
\hline 8.1 & 486 & 244 & 0.50 & 2.2 & 0.000523 & 0.16 & 193.30 & 2.71 & 0.0480 & 0.0018 & 0.0052 & 0.0001 & 33.2 & 0.5 \\
\hline 9.1 & 215 & 117 & 0.54 & 8.8 & - & 0.01 & 21.08 & 0.25 & 0.0523 & 0.0009 & 0.0474 & 0.0006 & 298.7 & 3.5 \\
\hline 10.1 & 245 & 173 & 0.70 & 4.4 & 0.000342 & $<0.01$ & 47.63 & 0.60 & 0.0486 & 0.0014 & 0.0210 & 0.0003 & 134.0 & 1.7 \\
\hline 11.1 & 56 & 35 & 0.62 & 0.3 & - & 1.66 & 166.89 & 4.95 & 0.0599 & 0.0058 & 0.0059 & 0.0002 & 37.9 & 1.2 \\
\hline 12.1 & 87 & 44 & 0.50 & 1.5 & 0.001569 & 0.42 & 48.56 & 0.81 & 0.0520 & 0.0022 & 0.0205 & 0.0003 & 130.9 & 2.2 \\
\hline 13.1 & 339 & 95 & 0.28 & 1.4 & 0.003154 & 0.56 & 212.89 & 3.50 & 0.0510 & 0.0024 & 0.0047 & 0.0001 & 30.0 & 0.5 \\
\hline 14.1 & 48 & 24 & 0.50 & 0.3 & 0.005071 & 0.79 & 159.46 & 4.88 & 0.0531 & 0.0053 & 0.0062 & 0.0002 & 40.0 & 1.2 \\
\hline 15.1 & 198 & 103 & 0.52 & 0.8 & 0.002535 & 0.81 & 219.47 & 4.21 & 0.0530 & 0.0031 & 0.0045 & 0.0001 & 29.1 & 0.6 \\
\hline 16.1 & 49 & 31 & 0.63 & 0.3 & 0.005408 & 3.13 & 162.06 & 5.02 & 0.0716 & 0.0062 & 0.0060 & 0.0002 & 38.4 & 1.2 \\
\hline 17.1 & 43 & 29 & 0.66 & 0.2 & 0.016669 & 1.55 & 174.17 & 6.00 & 0.0590 & 0.0066 & 0.0057 & 0.0002 & 36.3 & 1.3 \\
\hline 18.1 & 281 & 68 & 0.24 & 1.1 & 0.003152 & 5.36 & 227.23 & 3.96 & 0.0890 & 0.0042 & 0.0042 & 0.0001 & 26.8 & 0.5 \\
\hline 19.1 & 416 & 104 & 0.25 & 1.6 & - & 0.27 & 225.97 & 3.50 & 0.0488 & 0.0022 & 0.0044 & 0.0001 & 28.4 & 0.4 \\
\hline 20.1 & 195 & 215 & 1.10 & 0.8 & 0.000318 & 0.93 & 221.83 & 4.48 & 0.0539 & 0.0037 & 0.0045 & 0.0001 & 28.7 & 0.6 \\
\hline
\end{tabular}

Uncertainties given at the one $\sigma$ level. $\mathrm{f}_{206} \%$ denotes the percentage of ${ }^{206} \mathrm{~Pb}$ that is common $\mathrm{Pb}$.

characteristics resemble those of the La Junta Formation (Urbina, 2001). According to Encinas et al. (2014), the La Junta Formation may have been deposited during the initial stages of subsidence that rapidly led to marine ingression and deposition of the siltstone, fossiliferous black shale and fine-grained sandstone of the Vargas Formation (Urbina, 2001). These marine strata are highly deformed and foliated, and sedimentary structures are not preserved. Nevertheless, their lithological association, with a high content of organic matter as indicated by their black color, the occurrence of poorly preserved gastropods, bivalves, echinoderms and planktonic foraminifera, plus the common occurrence of pyrite, are all features that resemble those exhibited by the "Siltstone and Very Fine-Grained Sandstone" sedimentary facies defined by Encinas et al. (2013) for a portion of the upper member of the Ayacara Formation.

Despite the limited number of analyses comprising the age spectra reported here, there is a marked difference between the sedimentary rocks of the
Traiguén Formation provenance in the Puerto Cisnes-Queulat area and the results obtained by Hervé et al. (2001) and Encinas et al. (2016a) around its type locality (Table 2). In our study area, there is a relative absence of detrital zircon older than Late Cretaceous (except for a small number of Early Cretaceous and Permian ages), whereas the Traiguén Formation to the south shows a marked presence of Early Cretaceous and older reworked zircon derived from the metamorphic complexes, with source areas to the west and east as suggested by Encinas et al. (2016a). At the latitude of Queulat it seems that Cenomanian zircons represent the only significant pre-Cenozoic zircon population, presumably derived from plutonic rocks of this age in the North Patagonian Batholith immediately to the east (Pankhurst et al., 1999; Urbina, 2001; De la Cruz and Cortés, 2011). Similar detrital zircon age peaks are recognized in the La Junta and Vargas formations (this work; Encinas et al., 2014), and in the lower member of the Ayacara Formation (Encinas et al., 2013) (Table 2), 
TABLE 2. SUMMARY OF PUBLISHED U-Pb ZIRCON AGES OF EOCENE TO EARLY MIOCENE MARINE AND METAMORPHIC UNITS OF THE CHILEAN NORTH PATAGONIAN ANDES. BOLD LETTERS CORRESPOND TO PALEOGENE AGES.

\begin{tabular}{|c|c|c|c|c|c|c|}
\hline Formation/Unit & $\begin{array}{l}\text { Stratigraphic } \\
\text { age }\end{array}$ & Sample & Lithology-Protolith & $\begin{array}{c}\text { Maximum } \\
\text { depositional } \\
\text { age }\end{array}$ & $\begin{array}{c}\text { Main detrital zircon age } \\
\text { peaks }\end{array}$ & References \\
\hline \multirow{2}{*}{$\begin{array}{l}\text { Llancahué epi- } \\
\text { metamorphic } \\
\text { complex }\end{array}$} & \multirow{2}{*}{$\begin{array}{l}\text { Early Eocene- } \\
\text { Oligocene }\end{array}$} & FO14158 & Quartzite - Tuff & $53 \mathrm{Ma}$ & - & \multirow{3}{*}{ Hervé et al., 2017} \\
\hline & & FO14163 & Metasandstone & $36 \mathrm{Ma}$ & ca. 50, $375 \mathrm{Ma}$ & \\
\hline \multirow{6}{*}{ Ayacara Fm. } & \multirow{6}{*}{$\begin{array}{l}\text { Lower to Middle } \\
\text { Miocene }\end{array}$} & FO14168 & Tuffaceous sandstone & $22 \mathrm{Ma}$ & - & \\
\hline & & CAYA 4 & Sandstone & $18 \mathrm{Ma}$ & ca. $20,121-132,303 \mathrm{Ma}$ & \multirow{5}{*}{ Encinas et al., 2013} \\
\hline & & CAYA 14 & Sandstone & $22 \mathrm{Ma}$ & ca. $23 \mathrm{Ma}$ & \\
\hline & & CAYA 24 & Sandstone & $18 \mathrm{Ma}$ & ca. 21-23 Ma & \\
\hline & & IMAN 14 & Sandstone & $19 \mathrm{Ma}$ & ca. $20 \mathrm{Ma}$ & \\
\hline & & IMAN 20 & Sandstone & $20 \mathrm{Ma}$ & ca. 21-22 Ma & \\
\hline \multirow{2}{*}{ Puduhuapi Fm. } & \multirow{2}{*}{$\begin{array}{l}\text { Late Oligocene- } \\
\text { Early Miocene }\end{array}$} & PUD-1 & Sandstone & $23 \mathrm{Ma}$ & - & \multirow{6}{*}{ Encinas et al., 2014} \\
\hline & & PUD-7 & Sandstone & $23 \mathrm{Ma}$ & - & \\
\hline \multirow{2}{*}{ La Cascada Fm. } & \multirow{2}{*}{$\begin{array}{l}\text { Early-Middle } \\
\text { Miocene }\end{array}$} & PNEG & Sandstone & $49 \mathrm{Ma}$ & $c a .84 \mathrm{Ma}$ & \\
\hline & & NONO-2 & Sandstone & $18 \mathrm{Ma}$ & ca. $110-117 \mathrm{Ma}$ & \\
\hline \multirow{2}{*}{ Vargas Fm. } & \multirow{2}{*}{$\begin{array}{l}\text { Late Oligocene- } \\
\text { Early Miocene }\end{array}$} & VAR-2 & Sandstone & $39 \mathrm{Ma}$ & ca. $82,93 \mathrm{Ma}$ & \\
\hline & & VAR-5 & Sandstone & $84 \mathrm{Ma}$ & ca. $109 \mathrm{Ma}$ & \\
\hline \multirow{2}{*}{ La Junta Fm. } & \multirow{2}{*}{$\begin{array}{l}\text { Late Oligocene- } \\
\text { Early Miocene }\end{array}$} & FO1101 & Paraconglomerate (matrix) & $28 \mathrm{Ma}$ & ca. $98 \mathrm{Ma}$ & This work \\
\hline & & JUN-1 & Sandstone & $26 \mathrm{Ma}$ & ca. $96-108 \mathrm{Ma}$ & Encinas et al., 2014 \\
\hline \multirow{11}{*}{ Traiguén Fm. } & \multirow{2}{*}{$\begin{array}{l}\text { Late Oligocene- } \\
\text { Early Miocene }\end{array}$} & FO1104 & $\begin{array}{l}\text { Metaconglomerate } \\
\text { (matrix) }\end{array}$ & 26/29 Ma & ca. 35, 39, $96 \mathrm{Ma}$ & \multirow[t]{2}{*}{ This work } \\
\hline & & FO1105 & Schist & $28 \mathrm{Ma}$ & ca. 39 Ма & \\
\hline & Early Oligocene & $\begin{array}{l}\text { Isla Mita- } \\
\text { hues } 1.1\end{array}$ & Volcanic breccia & $32 \mathrm{Ma}$ & - & \multirow{8}{*}{$\begin{array}{l}\text { Encinas et al., } \\
\text { 2016a }\end{array}$} \\
\hline & \multirow{8}{*}{$\begin{array}{l}\text { Late Oligocene- } \\
\text { Early Miocene }\end{array}$} & $\begin{array}{l}\text { Las Men- } \\
\text { tas } 1.1\end{array}$ & Lapilli tuff & $23.4 \mathrm{Ma}$ & - & \\
\hline & & $\begin{array}{l}\text { Las Men- } \\
\text { tas } 3.5\end{array}$ & Sandstone & $23 \mathrm{Ma}$ & ca. $292 \mathrm{Ma}$ & \\
\hline & & $\begin{array}{l}\text { Las Men- } \\
\text { tas } 12.1\end{array}$ & Sandstone & $26 \mathrm{Ma}$ & $\begin{array}{l}\text { ca. } 126,276,470,700- \\
720,1,006 \mathrm{Ma}\end{array}$ & \\
\hline & & $\begin{array}{l}\text { Isla Ana } \\
1.1\end{array}$ & Sandstone & $127 \mathrm{Ma}$ & $\begin{array}{l}\text { ca.287, 256-477, 574, } \\
1,086 \mathrm{Ma}\end{array}$ & \\
\hline & & $\begin{array}{l}\text { Isla Rojas } \\
1.1\end{array}$ & Sandstone & $25 \mathrm{Ma}$ & $\begin{array}{l}\text { ca. 127, 272, 382-407, } \\
467-554,1,055-1,099 \mathrm{Ma}\end{array}$ & \\
\hline & & $\begin{array}{l}\text { Isla Rojas } \\
6.5\end{array}$ & Sandstone & $128 \mathrm{Ma}$ & $\begin{array}{l}\text { ca. 252-289, 359-408, } \\
476,525-582,1,075 \mathrm{Ma}\end{array}$ & \\
\hline & & $\begin{array}{l}\text { McPher- } \\
\text { son } 2.2\end{array}$ & Sandstone & $126 \mathrm{Ma}$ & $\begin{array}{l}\text { ca. } 267,390,477,1,050- \\
1,085 \mathrm{Ma}\end{array}$ & \\
\hline & & IT983 & Sandstone & $26 \mathrm{Ma}$ & ca. $68-72,>250 \mathrm{Ma}$ & Hervé et al., 2001 \\
\hline
\end{tabular}

suggesting the existence of an emerged ridge of plutonic rocks marking the eastern border of the marine basins. Colwyn et al. (2019) also postulate the existence of a topographic barrier similar to the modern mountain range, to explain a rain shadow in extra-Andean Patagonia indicated by $\delta \mathrm{D}$ isotope data, at least since Paleocene times.

\subsection{Age constraints on the metamorphism of the Traiguén Formation in the Puerto Cisnes- Queulat area}

Even though no radiometric ages are reported here for the metamorphism of the studied rocks, some limits may be inferred if we take into account its relationship 
to Neogene magmatism in the area (Fig. 2). Plutonic rocks in the Queulat-Puerto Cisnes-Isla Magdalena area have been dated by various geochronological methods (Hervé et al., 1993; Pankhurst et al., 1999; Parada et al., 2000; Cembrano et al., 2002; Mella and Duhart, 2011), yielding results between 10 and $20 \mathrm{Ma}$. The most voluminous intrusive rocks in the Queulat area correspond to the dioritic and tonalitic facies of the Queulat Diorites (informal name) of Parada et al. (2000), who report that they intrude "highly deformed metapelites of unknown age". The latter rocks are here confirmed as belonging to the Traiguén Formation. ${ }^{40} \mathrm{Ar} /{ }^{39} \mathrm{Ar}$ ages of $16-18 \mathrm{Ma}$ in amphibole of the Queulat Diorites are consistent with the $c a$. $15 \mathrm{Ma} \mathrm{Rb-Sr}$ whole-rock isochron obtained by Pankhurst et al. (1999) in granodiorite and tonalite at Seno Ventisquero (north of Queulat, Fig. 2), and with the $c a$. 14.5 Ma hornblende and biotite ${ }^{40} \mathrm{Ar} /{ }^{39} \mathrm{Ar}$ ages obtained by Cembrano et al. (2002) for a non-deformed diorite sampled in the same area. There is also a $15.2 \pm 0.19$ Ma SHRIMP $\mathrm{U}-\mathrm{Pb}$ crystallization age for the Cerro Esternón amphibole \pm biotite tonalite (Fig. 2), a stock located just south of Puerto Cisnes (Mella and Duhart, 2011). To the west, at Isla Magdalena, Pankhurst et al. (1999) obtained consistent $c a$. $20 \mathrm{Ma} \mathrm{Rb-Sr}$ wholerock isochrons for biotite granodiorite intruding the Traiguén Formation. These geochronological data attest to a relatively significant episode of magma emplacement during 20-10 Ma, particularly between 20-15 Ma. Plutonic rocks from this episode intrude the Main Range Metamorphic Complex, as well as the Cretaceous rocks of the batholith and the metasedimentary rocks of the Traiguén Formation and suggest a minimum age for the regional metamorphism affecting the latter.

Randomly orientated biotite micro-porphyroblasts in the Puerto Cisnes schist were generated by contact metamorphism and postdate the banding and crenulation cleavage formed during regional metamorphism. Parada et al. (2000) describe the garnet-biotite-staurolite-andalusite-sillimanite mineral paragenesis in nearby pelitic hornfels, deducing a $3 \mathrm{kbar}$ maximum pressure for the contact metamorphism. Hervé et al. (1993) reported a similar pressure of emplacement for the Rio Cisnes leucogranite stock, which intruded the metamorphic rocks of the Traiguén Formation at $10 \mathrm{Ma}$ (Mella and Duhart, 2011). This relationship implies that the Traiguén Formation was already folded and metamorphosed before intrusion of the Río Cisnes stock and generation of the contact metamorphic aureole.

At the north-eastern tip of Isla Refugio (Fig. 2),

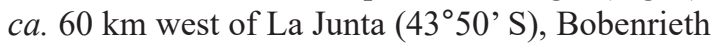
et al. (1983) assigned a package of volcanic rocks to the Traiguén Formation, some of them with preserved pillow structures (Fig. $3 \mathrm{H}$ ). These rocks grade into mylonitic schists in a $2 \mathrm{~km}$ long west-toeast transect. The main foliation is approximately NNE and dips $70^{\circ}-80^{\circ} \mathrm{E}$, with a dextral sense of shear (Fig. 3G), resembling the modern kinematics of the LOFZ. Post-tectonic microdiorite and aplite dykes were emplaced parallel to the main foliation. Thus, the timing of deformation should be older than $15.4 \pm 0.5 \mathrm{Ma}$, the whole-rock/biotite $\mathrm{Rb}-\mathrm{Sr}$ isochron age obtained by Pankhurst et al. (1999) in a non-deformed suite of granodiorites, microdiorite and aplite mapped as intruding the rocks of the Traiguén Formation on the same island (Fig. 2). Late Miocene LOFZ-related mylonites have been recognized in the study area (e.g., Cembrano et al., 2002), and a previous early-to-mid Miocene event of deformation and metamorphism is recorded by the data presented here.

The Cenozoic growth of the North Patagonian Andes between $45^{\circ} \mathrm{S}$ and $47^{\circ} \mathrm{S}$ has been recently addressed by Folguera et al. (2018a). During this Era, they identified a discrete pulse of contraction at $c a$. 16-18 Ma on the basis of U-Pb detrital zircon ages from synorogenic strata located to the east of the Cretaceous outcrops of the North Patagonian Batholith. To the east of Queulat, in the Alto Río Cisnes area, a tuff interleaved with fluvial syntectonic strata of the Río Frías Formation has yielded a 15.8 Ma U-Pb zircon age (Encinas et al., 2016b), confirming the early-mid Miocene lateral expansion of the Patagonian Andes at these latitudes. These ages are similar to the above-mentioned $c a$. 15-20 Ma crystallization ages for the batholith in the Queulat area, and are in agreement with the beginning of transpression along the LOFZ at $c a$. 16 Ma deduced from fission track ages (Thomson, 2002). Parada et al. (2000) suggest that the diorites and tonalites of the Queulat area were produced by melting of the lower crust at $c a .33 \mathrm{~km}$ depth. Such deep partial melting was ascribed to high exhumation rates (between 1-2 $\mathrm{mm} / \mathrm{yr}$ ) due to vertical movement along the LOFZ. Hervé et al. (1993) explained the formation of the Río Cisnes leucogranite in a similar way. In this scenario, tectonic inversion of the Traiguén Basin in 
the Queulat area would have occurred during early to middle Miocene times, roughly coeval with the early development of the continental foreland system to the east and major batholith emplacement in the Main Cordillera.

During these processes, tectonic emplacement and metamorphism of ultramafic bodies occurred around the Llancahué-Ayacara area (Hervé et al., 2017; González, 2018) and also to the south, in Isla Traiguén and Isla Magdalena, as shown by N-S trending positive gravity gradient anomalies (Folguera et al., 2018b). These ultramafic bodies may be a proxy for the amount of extension generated during opening of these basin and the depth of the detachment fault during their closure.

\subsection{Paleogene magmatism along the LOFZ?}

The U-Pb detrital zircon ages reported in this contribution together with previously published data for the late Oligocene to early Miocene marine sedimentary rocks (Table 2), allows a N-S comparison of the basins located close to the LOFZ between $42^{\circ} \mathrm{S}$ and $46^{\circ} \mathrm{S}$ (Hervé et al., 2001; Encinas et al., 2013; Encinas et al., 2014; Encinas et al., 2016a; Hervé et al., 2017). Sediment provenance here is important as an indirect evidence of magmatic evolution, since the outcrop record is limited due to large-scale denudation during late Cenozoic times (Thomson, 2002; Adriasola et al., 2005) and the dense vegetation cover of the area. The distribution of detrital zircon ages shows main relative probability peaks of early Miocene ( $c a .23-17 \mathrm{Ma}$ ), late Oligocene ( $c a .28-25 \mathrm{Ma}$ ), late Eocene-early Oligocene ( $c a$. 39-32 Ma) and early Eocene ( $c a$. 53-50 Ma). Although the early Neogene peaks might correspond to now-eroded early Miocene extrusive equivalents of the North Patagonian Batholith, the source of the Paleogene detrital zircons is less obvious. Paleogene magmatic rocks are found hundreds of $\mathrm{km}$ to the east in the Pilcaniyeu and El Maitén volcanic belts along the western edge of the North Patagonian Massif (e.g., Rapela et al., 1988; Iannelli et al., 2017; Fernández Paz et al., 2019) and, more restrictedly, tens of $\mathrm{km}$ east in the Alto Rio Císnes area (Prieto and Cortés, 1995; De la Cruz and Cortés, 2011) and the Meseta Guadal area (Encinas et al., 2019). These are potential sources for the Paleogene detrital zircons, however, the likely existence of an emerged ridge to the east of the basins, the immature nature of some of the protoliths and sedimentary rocks (e.g., the Queulat metaconglomerate and the La Junta Formation) and the occurrence of scattered Paleogene magmatic rocks along the LOFZ, all favour the possibility of a more local derivation, as discussed below.

Basaltic pillow lavas have been the most studied components of the Traiguén Formation volcanism, but this unit also includes intermediate to felsic igneous rocks. Bobenrieth et al. (1983) and Hervé et al. $(1994,1995)$ refer to the existence of felsic tuffs, dacite lava flows and porphyritic dacites and rhyolites, particularly on Isla Magdalena. Bobenrieth et al. (1983) and Urbina (2001) also indicate the existence of andesitic lava flows interdigitated with the sedimentary rocks of the La Junta Formation. This intermediate to acid volcanism is a plausible local source for the abundant supply of 28-25 Ma zircons recorded in the Traiguén and La Junta formations.

Late Eocene-early Oligocene (39-32 Ma) detrital zircons are abundant in the rocks sampled, as well as in those studied by Hervé et al. (2017) on Isla Llancahué, where they reported a late Eocene maximum possible sedimentation age for a foliated metasandstone with graded bedding, interleaved with black slate showing slump structures. At Llancahué, the metasedimentary rocks are related to and (most probably overlie) pillow metabasalts with a common foliation (Hervé et al., 2017)-a lithological association that resembles that of the Traiguén Formation. This age compares with that of early Oligocene volcanism recognized on Isla Mitahués (Encinas et al., 2016a) and with the $\mathrm{K}-\mathrm{Ar}$ age peak population of $c a .34 \mathrm{Ma}$ reported by Herrera (2000) for the dyke swarm intruding the rocks of the Traiguén Formation. In addition, late Eocene plutonic rocks are also spatially related to both the Traiguén Formation and the LOFZ in the archipelagos of Aysén (Pankhurst et al., 1999).

Paleocene to early Eocene detrital zircons ages have not been found in the study area and their occurrence is restricted to the Llancahue epimetamorphic complex, from a metamorphosed tuff interleaved with micaschist, metasandstones and minor metabasites (Hervé et al., 2017). The only known plutonic rocks of similar age in the area are located close to the LOFZ around Lago Yelcho ( $c a .43^{\circ} 20^{\prime} \mathrm{S}$ ) and have K-Ar ages of 50-55 Ma (Duhart, 2008).

Thus, sporadically, and spatially distributed Paleogene magmatism was focalized along structures 
corresponding to the modern LOFZ, suggesting a zone of crustal weakness prior to Neogene times (similarly to the Proto Liquiñe-Ofqui fault of Aragón et al., 2011).

\subsection{Tectonic context}

Herve (1976) was the first to propose ancient displacements along the LOFZ based on the existence of undeformed dykes crosscutting mylonites near Liquiñe $\left(\mathrm{ca} .39^{\circ} \mathrm{S}\right)$ and interpreted the former as Late Cretaceous to early Cenozoic in age by correlation with dated intrusive rocks cropping out in the area. Later studies dated various lithologies of hypabyssal intrusives with similar crosscutting relationships elsewhere in the Liquiñe area, obtaining pre-Neogene ages of $29 \mathrm{Ma}$ (whole rock K-Ar by Hervé et al., 1979), $48 \mathrm{Ma}\left({ }^{40} \mathrm{Ar} /{ }^{39} \mathrm{Ar}\right.$ biotite stepheating by Schermer et al., 1996) and $100 \mathrm{Ma}$ $\left({ }^{40} \mathrm{Ar}{ }^{39} \mathrm{Ar}\right.$ amphibole step-heating by Cembrano et al., 2000). In accordance with this, Bourguois et al. (2016) infer that the inception of the Golfo de
Penas basin, whose evolution is controlled by the LOFZ, is represented by the deposition of the marine beds of the mid-Eocene Puerto Good Sequence (Forsythe et al., 1985).

The new data and synthesis presented in this work argue in favour of the LOFZ exerting structural control on Cenozoic magma emplacement and basin formation. The fault system would have been reactivated with kinematics appropriate to the prevailing subduction parameters (e.g., Cembrano et al., 2000). In Paleogene times the low obliquity and slow subduction of the Farallon plate would have promoted margin-parallel transtensional tectonics (Hervé et al., 1995; Aragón et al., 2011) leading to the formation of a chain of small volcano-sedimentary basins during the northward drift of the peninsular coastal block (or the Chiloé block in Fig. 5), in accordance with the buttressing model of Beck et al. (1993). On the other hand, the faster and nearly orthogonal late Oligocene to early Miocene subduction of the Nazca plate (Fig. 5) led to vigorous slab roll-back (e.g., Muñoz et al., 2000; Fennell et al., 2018), enhanced stretching in

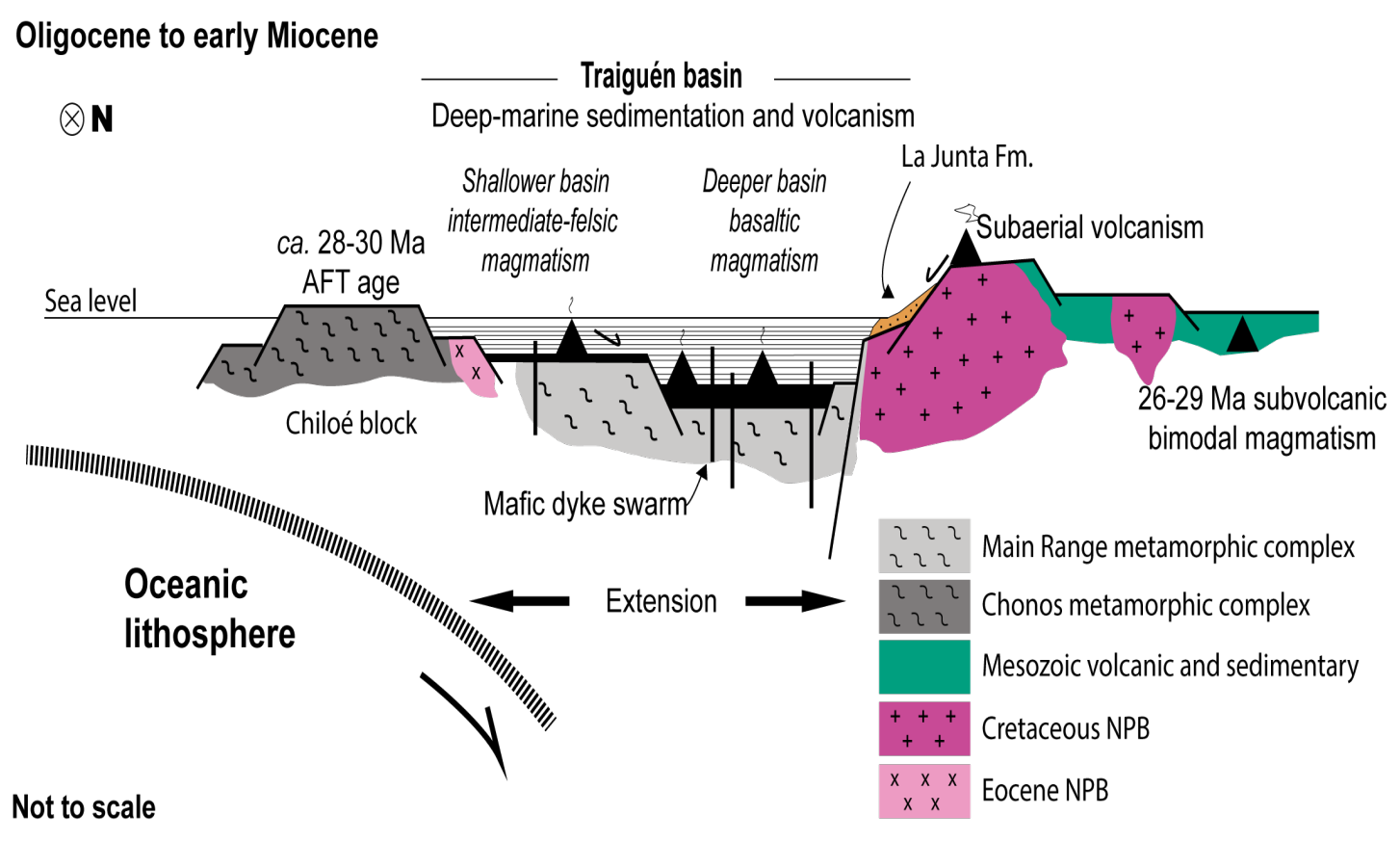

FIG. 5. Schematic E-W geological cross-section through the Queulat area showing paleogeographic reconstruction during Oligocene to early Miocene times based on data and interpretations summarized in the text. AFT: Apatite fission tracks ages after Thomson et al. (2001). Oceanic-like mafic crust was emplaced in zones of maximum extension. Basin depth variations after Hervé et al. (1994). NPB: North Patagonian Batholith. 
the upper plate and widespread magmatism in the forearc to the backarc (Muñoz et al., 2000; Encinas et al., 2016a; Ianelli et al., 2017; Fernandez Paz et al., 2019 and references therein). The climax of the extension is recorded in the southern sectors of the LOFZ, at these latitudes it is thought that the Chiloé block was detached from the continent during the retreat of the trench towards the ocean, then colliding against the stable continent during the middle Miocene (Hervé et al., 2017).

\section{Conclusion}

The $c a .28$ Ma youngest detrital zircons ages obtained from the low-grade metamorphic rocks of Puerto Cisnes-Queulat area clearly indicate that the protoliths were deposited during Rupelian-Chattian, precluding correlation with the Paleozoic rocks of the Main Range metamorphic complex to the west, but validating correlation with the metasedimentary rocks of the Traiguén Formation, as previously suggested by Bobenrieth et al. (1983). Similar detrital zircon age patterns in rock samples from the La Junta and Traiguén formations suggest that the former represents a high energy lateral facies change of the latter, deposited in a basin -or basins- bounded to the east by an emerged ridge of Cretaceous plutonic rocks (Fig. 5).

The wide areal distribution of metamorphic rocks with protoliths of early-to-mid Cenozoic age in a similar position with respect to the main lineament of the LOFZ (Hervé et al., 2017) suggests a regional pattern of early Neogene deformation and metamorphism associated with the closure of the intra-arc basin, or basins, located in the western slope of the North Patagonian Andes.

Even though marine ingressions covered wide areas of southern South America during late Oligoceneearly Miocene times as result of regional extension (see Encinas et al., 2018 for review), deep-marine sedimentation together with the generation of quasioceanic crust is restricted to the LOFZ domain (Hervé et al., 1995; Silva et al., 2003; Hervé et al., 2017). Although more studies should be carried out to understand the associated mechanisms in detail, the crustal scale discontinuity represented by the LOFZ appears to have played an important role during early-to-mid Cenozoic magmatic activity, and the opening and subsequent closure of basins in the western slope of North Patagonian Andes.

\section{Acknowledgements}

Fondecyt projects 1080516 (M. Suárez) and 1095099 (F. Hervé) financed the field work, and project 1130227 (F. Hervé) the analytical work. The Fondecyt grant 1180457 (F. Hervé) provided time and impetus to produce the present paper. P. Quezada thanks to N. López, F. Reyes and E. Crisostomo for their help during part of the field work. Revisions by Drs. S. Oriolo and L. Fennell improved the paper.

\section{References}

Adriasola, A.C.; Thomson, S.N.; Brix, M.R.; Hervé, F.; Stockhert, B. 2005. Postmagmatic cooling and late Cenozoic denudation of the North Patagonian Batholith in the Los Lagos region of Chile, $41^{\circ}-42^{\circ} 15^{\prime} \mathrm{S}$. International Journal of Earth Sciences 95: 504. doi: 10.1007/s00531-005-0027-9.

Aragón, E.; D`Eramo, F.; Castro, A.; Pinotti, L.; Brunelli, D.; Rabbia, O.; Rivalenti, G.; Varela, R.; Spackman, W.; Demartis, M.L.; Cavarozzi, C.E.; Aguilera, Y.; Mazzucchelli, M.; Ribot, A. 2011. Tectono-magmatic response to major convergence changes in the north Patagonian suprasubduction system: the Paleogene subduction-transcurrent plate margin transition. Tectonophysics 509: 218-237.

Aragón, E.; Pinotti, L.; D’Eramo, F.; Castro, A.; Rabbia, O.; Coniglio, J.; Demartis, M.; Hernando, I.; Cavarozzi, C.E.; Aguilera, Y.E. 2013. The Farallon-Aluk ridge collision with South America: Implications for the geochemical changes of slab window magmas from fore-to back-arc. Geoscience Frontiers 4: 377-388.

Bechis, F.; Encinas, A.; Concheyro, A.; Litvak, V.D.; Aguirre-Urreta, B.; Ramos, V.A. 2014. New age constraints for the Cenozoic marine transgressions of northwestern Patagonia, Argentina $\left(41^{\circ}-43^{\circ} \mathrm{S}\right)$ : Paleo-geographic and tectonic implications. Journal of South American Earth Sciences 52: 72-93.

Beck, M.; Rojas, C.; Cembrano, J. 1993. On the nature of buttressing in strike slip fault systems. Geology 21: 755-758.

Bobenrieth, L.; Díaz, F.; Davidson, J.; Portigliati, C. 1983. Complemento al Mapa Metalogénico XI Región, Sector Norte continental comprendido entre 45' latitud Sur y el límite con la X Región. Informe (Inédito), Servicio Nacional de Geología y Minería: 154 p. Santiago.

Bourgois, J.; Lagabrielle, Y.; Martin, H.; Dyment, J.; Frutos, J.; Cisternas, M.E. 2016. A review on forearc 
ophiolite obduction, Adakite-like generation, and slab window development at the Chile triple junction area: uniformitarian framework for spreading-ridge subduction. Pure and Applied Geophysics 173: 3217 3246. doi: 10.1007/s00024-016-1317-9.

Cande, S.C.; Leslie, R.B. 1986. Late Cenozoic tectonics of the Southern Chile trench. Journal of Geophysical Research 91 (B1): 471-496.

Cembrano, J.; Hervé, F.; Lavenu, A. 1996. The LiquiñeOfqui Fault Zone: a long lived intra-arc fault system in southern Chile. Tectonophysics 250: 55-66.

Cembrano, J.; Schermer, E.; Lavenu, A.; Sanhueza, A. 2000. Contrasting nature of deformation along an intra-arc shear zone, the Liquiñe-Ofqui fault zone, southern Chilean Andes. Tectonophysics 319: 129-149.

Cembrano, J.; Lavenu, A.; Reynolds, P.; Arancibia, G.; López, G.; Sanhueza, A. 2002. Late Cenozoic transpressional ductile deformation north of the Nazca-South America-Antarctica triple junction. Tectonophysics 354: 289-314.

Colwyn, D.A.; Brandon, M.T.; Hren, M.T.; Hourigan, J.; Pacini, A.; Cosgrove, M. G.; Midzic, M.; Garreau, R.; Metzger, C. 2019. Growth and steady state of the Patagonian Andes. American Journal of Science 319 (6): 431-472. doi: 10.2475/06.2019.01.

Cuitiño, J.; Dozo, M.T.; del Río, C.J.; Buono, M.R.; Palazzesi, L.; Fuentes, S.; Scasso, R. 2017. Miocene Marine Transgressions: Paleoenvironments and Paleobiodiversity. In Late Cenozoic of Península Valdés, Patagonia, Argentina (Bouza, P.; Bilmes, A.; editors). Springer Earth System Sciences: 47-84. doi: 10.1007/978-3-319-48508-9 3.

Davidson, J.; Mpodozis, C.; Godoy, E.; Hervé, F.; Pankhurst, R.J.; Brook, M. 1987. Late Paleozoic accretionary complexes on the Gondwana margin of southern Chile: evidence from the Chonos Archipelago. In Gondwana Six: Structure, Tectonics and Geophysics (McKenzie, G.D.; editor). Geophysical Monograph, American Geophysical Union 40: 221-227.

De La Cruz, R.; Cortés, J. 2011. Geología del área oriental de la Hoja Puerto Cisnes, Región Aysén del General Carlos Ibáñez del Campo. Servicio Nacional de Geología y Minería, Carta Geológica de Chile, Serie Geología Básica 127: 70 p., 1 mapa escala 1:250.000.

Dickinson, W.R.; Gehrels, G.E. 2009. Use of U-Pb ages of detrital zircons to infer maximum depositional ages of strata: a test against a Colorado Plateau Mesozoic database. Earth and Planetary Science Letters 288 (1-2): 115-125.
Duhart, P. 2008. Processos metalogenéticos em ambientes de arco magmático tipo andino, caso de estudo: mineralizacoes da regiao dos Andes Patagonicos setentrionais do Chile. Tesis de Doutorado (Unpublished), Universidade de Sao Paulo, Instituto de Geociencias: 215 p. Brasil.

Eagles, G.; Scott, B.G. 2014. Plate convergence west of Patagonia and the Antarctic Peninsula since $61 \mathrm{Ma}$. Global and Planetary Change 123: 189-198.

Encinas, A.; Zambrano, P.A.; Finger, K.L.; Valencia, V.; Buatois, L.A.; Duhart, P. 2013. Implications of Deepmarine Miocene Deposits on the Evolution of the North Patagonian Andes. Journal of Geology 121: 215-238.

Encinas, A.; Pérez, F.; Nielsen, S.N.; Finger, K.L.; Valencia, V.; Duhart, P. 2014. Geochronologic and paleontologic evidence for a Pacific-Atlantic connection during the late Oligocene-early Miocene in the Patagonian Andes (43-44 S). Journal of South American Earth Sciences 55: 1-18.

Encinas, A.; Folguera, A.; Oliveros, V.; De Girolamo Del Mauro, L.; Tapia, F.; Riffo, R.; Hervé, F.; Finger, K.L.; Valencia, V.A.; Gianni, G.; Álvarez, O. 2016a. Late Oligocene-early Miocene submarine volcanism and deep-marine sedimentation in an extensional basin of southern Chile: implications on the tectonic development of the North Patagonian Andes. Geological Society of America Bulletin 128 (5-6): 807. doi: 10.1130/B31303.1.

Encinas, A.; Folguera, A.; Litvak, V.D.; Echaurren, A.; Gianni, G.; Fernández Paz, F.; Bobe, R.; Valencia, V. 2016b. New age constraints for the Cenozoic deposits of the Patagonian Andes and the Sierra de San Bernardo between $43^{\circ}$ and $46^{\circ} \mathrm{S}$. In Simposio de tectónica sudamericana, No. 1: p. 140. Santiago.

Encinas, A.; Folguera, A.; Bechis, F.; Finger, K.L.; Zambrano, P.; Pérez, F.; Bernabé, P.; Tapia, F.; Riffo, R.; Buatois, L.; Orts, D.; Nielsen, S.N.; Valencia, V.; Cuitiño, J.; Oliveros, V.; De Girolamo del Mauro, L.; Ramos, V. 2018. The Late Oligocene-Early Miocene Marine Transgression of Patagonia. In The Evolution of the Chilean-Argentinean Andes (Folguera, A.; Contreras Reyes, E.; Heredia, N.; Encinas, A.; Iannelli, S.; Oliveros, V.; Dávila, F.; Collo, G.; Giambiagi, L.; Maksymowicz, A.; Iglesia Llanos, M.P.; Turienzo, M.; Naipauer, M.; Orts, D.; Litvak, V.; Alvarez, O.; Arriagada, C.; editors). Springer Earth System Sciences. Springer, Cham.: 443-474. doi: 10.1007/978-3-31967774-3_18.

Encinas, A.; Folguera, A.; Riffo, R.; Molina, P.; Fernández Paz, L.; Litvak, V.D; Colwyn, D.A.; Valencia, V.A.; Carrasco, M. 2019. Cenozoic basin evolution of the Central 
Patagonian Andes: Evidence from geochronology, stratigraphy, and geochemistry. Geoscience Frontiers 10 (3): 1139-1165. doi: 10.1016/j.gsf.2018.07.004.

Espinoza, F.; Morata, D.; Pelleter, E.; Maury, R.C.; Suárez, M.; Lagabrielle, Y.; Polvé, M.; Bellon, H.; Cotton, J.; De la Cruz, R.; Guivel, C. 2005. Petrogenesis of the Eocene and Mio-Pliocene alkaline basaltic magmatism in Meseta Chile Chico, southern Patagonia, Chile: evidence for the participation of two slab windows. Lithos 82: 315-343.

Espinoza, W.; Fuenzalida, P. 1971. Geología de las Hojas Isla Rivero, Puerto Aysén y Balmaceda entre los Paralelos $45^{\circ}$ y $46^{\circ}$ de Latitud Sur. Instituto de Investigaciones Geológicas Convenio Instituto CORFO Aysén: 50 p. Santiago

Fernández Paz, L.; Bechis, F.; Litvak, V.D.; Echaurren, A.; Encinas, A.; González, J.; Lucassen, F.; Oliveros, V.; Valencia, V.; Folguera, A. 2019. Constraints on trenchward arc migration and back-arc magmatism in the North Patagonian Andes in the context of Nazca plate rollback. Tectonics 38: 3794-3817. doi: 10.1029/2019TC005580.

Folguera, A.; Encinas, A.; Echaurren, A.; Gianni, G.; Orts, D.; Valencia, V.; Carrasco, G. 2018a. Constraints on the Neogene growth of the central Patagonian Andes at the latitude of the Chile triple junction $\left(45-47^{\circ} \mathrm{S}\right)$ using $\mathrm{U} / \mathrm{Pb}$ geochronology in synorogenic strata. Tectonophysics 744: 134-154. doi: 10.1016/j. tecto.2018.06.011.

Folguera, A.; Gianni, G.; Encinas, A.; Álvarez, O.; Orts, D.; Echaurren, A.; Litvak, V.D.; Navarrete, C.; Sellés, D.; Tobal, J.; Ramos, M.; Fennell, L.; Fernández Paz, L.; Giménez, M.; Martínez, P.; Ruiz, F.; Iannelli, S. 2018b. Neogene Growth of the Patagonian Andes. In The Evolution of the Chilean-Argentinean Andes (Folguera, A.; Contreras Reyes, E.; Heredia, N.; Encinas, A.; Iannelli, S.; Oliveros, V.; Dávila, F.; Collo, G.; Giambiagi, L.; Maksymowicz, A.; Iglesia Llanos, M.P.; Turienzo, M.; Naipauer, M.; Orts, D.; Litvak, V.; Alvarez, O.; Arriagada, C.; editors). Springer Earth System Sciences. Springer, Cham. doi: 10.1007/978-3-319-67774-3_19.

Forsythe, R.D.; Olsson, R.K.; Johnson, C.; Nelson, E.P. 1985. New stratigraphic and mi- cropaleontologic observations from the Golfo de Penas-Taitao basin, southem Chile. Revista Geologica de Chile 25-26: 3-12. doi: 10.5027/andgeoV12n2-3-a01.

Frassinetti, D.; Covacevich, V. 1999. Invertebrados fósiles marinos de la Formación Guadal (Oligoceno Superior-Mioceno Inferior) en Pampa Castillo, Región de Aysén, Chile. Servicio Nacional de Geología y Minería. Boletín 51: 1-96. Santiago.

Fennell, L.; Quinteros, J.; Iannelli, S.; Litvak, V.; Folguera, A. 2018. The role of the slab pull force in the late Oligocene to early Miocene extension in the Southern Central Andes $\left(27^{\circ}-46^{\circ} \mathrm{S}\right)$ : Insights from numerical modeling. Journal of South American Earth Sciences 87 (2): 174-187. doi: 10.1016/j.jsames.2017.12.012.

Fuenzalida, R.; Etchart, H. 1975. Geología del Territorio de Aysén Comprendido entre los $43^{\circ} 45^{\prime}$ y los $45^{\circ}$ Latitud Sur. Informe (Inédito), Instituto de Investigaciones Geológicas: 99 p. Santiago.

Gianni, G.M.; Dávila, F.; Echaurren, A.; Fennell, L.; Tobal, J.; Navarrete, C; Quezada, P.; Folguera, A.; Giménez, M. 2018. A geodynamic model linking Cretaceous orogeny, arc migration, foreland dynamic subsidence and marine ingression in southern South America. Earth-Science Reviews 185:437-462. doi: 10.1016/j.earscirev.2018.06.016.

González, M. 2018. Caracterización petrográfica de los cuerpos ultramáficos en Chiloé Continental, sur de Chile. Memoria de Título (Inédito), Universidad Andrés Bello: 77 p. Santiago.

Haller, M.; Lapido, O. 1980. El Mesozoico de la Cordillera Patagónica Central. Revista de la Asociación Geológica Argentina 35: 230-47.

Herrera, C. 2000. Caracterización petrográfica y geoquímica del enjambre de diques básicos en la costa de la región de Aisén, $44^{\circ}$ L.S.- $47^{\circ}$ L.S.: Implicancias petrogenéticas. Memoria de Título (Inédito), Universidad de Chile Departamento de Geología: 52 p.

Hervé, M. 1976. Estudio Geológico de la Falla LiquiñeReloncaví en el área de Liquiñe: antecedentes de un movimiento transcurrente. In Congreso Geológico Chileno, No. 1, Actas 1: B39-B56. Santiago.

Hervé, F.; Araya, E.; Fuenzalida, J.L.; Solano, A. 1979. Edades radiométricas y tectónica neógena en el sector costero de Chiloé continental, X Región. In Congreso Geológico Chileno, No. 2, Actas 1: F1-F8. Arica.

Hervé. F.; Pankhurst. R.J.; Drake, R.; Beck, M.; Mpodozis, C. 1993. Granite generation and rapid unroofing related to strike-slip faulting. Aysén, Chile. Earth and Planetary Science Letters 120: 375-386.

Hervé, F. 1994. The Southern Andes between $39^{\circ}$ and $44^{\circ}$ LS: the geological signature of a transpressive tectonic regime related to a magmatic arc. In Tectonics of the Southern Central Andes (Reutter, K.J.; Scheuber, E.; Wigger, P.J.; editors). Springer-Verlag: 243-248. Berlin.

Hervé, F.; Suárez, M.; De la Cruz, R.; Belmar, M. 1994. Los depósitos volcanosedimentarios de la cuenca extensional 
intracontinental cenozoica de Isla Magdalena, Aysén, Chile. In Congreso Geológico Chileno, No. 7, Actas 2: 825-829. Concepción.

Hervé, F.; Pankhurst, R.J.; Drake, R.; Beck, M. 1995. Pillow metabasalts in a mid-Tertiary extensional basin adjacent to the Liquiñe-Ofqui fault zone: the Isla Magdalena area, Aysén, Chile. Journal of South American Earth Sciences 8: 33-46.

Hervé, F.; Sanhueza, A.; Silva, C.; Pankhurst, R.J.; Fanning, C.M.; Campbell, H.; Crundwell, M. 2001. A Neogene age for Traiguén Formation, Aysén, Chile, as revealed by SHRIMP U-Pb dating of detrital zircons. In Simposio Sudamericano de Geología Isotópica, No. 3: 570-574. Pucón.

Hervé, F.; Fanning, C.M. 2001. Late Triassic detrital zircons in meta-turbidites of the Chonos Metamorphic Complex, southern Chile. Revista Geológica de Chile 28 (1): 91- 104. doi: 10.5027/andgeoV28n1-a05.

Hervé, F.; Fanning, C.M.; Pankhurst, R.J. 2003. Detrital zircon age patterns and provenance in the metamorphic complexes of Southern Chile. Journal of South American Earth Sciences 16: 107-123.

Hervé, F.; Fuentes, F.; Calderón, M.; Fanning, C.M.; Quezada, P.; Pankhurst, R.; Rapela, C. 2017. Ultramafic rocks in the North Patagonian Andes: is their emplacement associated with the Neogene tectonics of the Liquiñe-Ofqui Fault Zone? Andean Geology 44 (1): 1-16. doi: 10.5027/andgeoV44n1-a01.

Ianelli, S.; Litvak, V.D.; Fernández Paz, L.; Folguera, A.; Ramos, M.; Ramos, V. 2017. Evolution of Eocene to Oligocene arc-related volcanism in the North Patagonian Andes $\left(39-41^{\circ} \mathrm{S}\right)$, prior to the break-up of the Farallon plate. Tectonophysics 696-697 (31): $70-87$.

Kay, S.M.; Gorring, M.; Ramos, V. 2004. Magmatic sources, setting and causes of Eocene to Recent Patagonian plateau magmatism $\left(36^{\circ} \mathrm{S}\right.$ to $\left.52^{\circ} \mathrm{S}\right)$. Revista de la Asociación Geológica Argentina 59 (4): 556-568.

Levi, B.; Aguilar, A.; Fuenzalida, R. 1966. Reconocimiento geológico en las provincias Llanquihue y Chiloé. Instituto de Investigaciones Geológicas, Boletín 19: 45 p. Santiago.

Lonsdale, P. 2005. Creation of the Cocos and Nazca plates by fission of the Farallon plate. Tectonophysics 404 : 237-264.

López-Escobar, L.; Cembrano, J.; Moreno, H. 1995. Geochemistry and tectonics of the Chilean Southern Andes basaltic quaternary volcanism (37-46 $\mathrm{S})$. Revista Geológica de Chile 22 (2): 219-234. doi: 10.5027/andgeoV22n2-a06.
Ludwig, K.R. 2003. Using Isoplot/Ex, v.3. A Geochronological toolkit for Microsoft Excel 4. Berkeley Geochronology Center, Special Publication 5: 75 p. California.

Malumián, N.; Yáñez, C. 2011. The Late CretaceousCenozoic transgressions in Patagonia and the Fuegian Andes: Foraminifera, palaeoecology, and palaeogeography. Biological Journal of the Linnean Society 103: 269-288.

Mella, M.; Duhart, P. 2011. Mapa geología base área Puerto Cisnes, región de Aysén, escala 1:50.000. Servicio Nacional de Geología y Minería-Gobierno Regional de Aysén, Investigación geológica minera ambiental en Aysén (Estudio FNDR, Código BIP N 30036527-0). Informe Final I: Geología Base-Mapas.

Morata, D.; Demant, A.; De la Cruz, R.; Barbero, L.; Suárez, M. 2003. Late Oligocene Peralkaline Rhyolites related with bimodal Volcanism in the Eastern Patagonian Cordillera: evidences for an incipient Back-Arc Extensional Basin? In Congreso Geológico Chileno No. 10, CD-ROM. Concepción.

Muñoz, J.; Troncoso, R.; Duhart, P.; Crignola, P.; Farmer, L.; Stern, C.R. 2000. The relation of the mid-Tertiary coastal magmatic belt in south-central Chile to the late Oligocene increase in plate convergence rate. Revista Geológica de Chile 27 (2): 177-203. doi: 10.5027/andgeoV27n2-a03.

Pankhurst, R.J.; Hervé, F.; Rojas, L.; Cembrano, J. 1992. Magmatism and tectonics in continental Chiloé, Chile (42-42³0’ S). Tectonophysics 205: 283-294.

Pankhurst, R.; Weaver, S.; Hervé, F.; Larrondo, P. 1999. Mesozoic-Cenozoic evolution of the North Patagonian Batholith in Aysén, southern Chile. Journal of the Geological Society of London 156: 673-694. Parada, M.A.; Lahsen, A.; Palacios, C. 2000. The Miocene plutonic event of the Patagonian Batholith at 4430' S: Thermochronological and geobarometric evidence for melting of a rapidly exhumed lower crust. Transactions of the Royal Society of Edinburgh: Earth Sciences 91: 169-179.

Pardo Casas, F.; Molnar, P. 1987. Relative motion of the Nazca (Farallon) and South American plates since late Cretaceous time. Tectonics 6 (3): 233-248.

Prieto, X.; Cortés, J. 1995. Geología del sector Oriental de la Hoja Río Cisnes $\left(71^{\circ}\right.$ a $72^{\circ} 20^{\prime} \mathrm{W}$ y $44^{\circ}$ a $\left.45^{\circ} \mathrm{S}\right)$, Región de Aysén. Informe (Inédito). Servicio Nacional de Geología y Minería, Gobierno Regional XI Región: 50 p. Santiago.

Rapela, C.W.; Spalletti, L.A.; Merodio, J.C.; Aragón, E. 1988. Temporal evolution and spatial variation 
of early tertiary volcanism in the Patagonian Andes ( $\left.40^{\circ} \mathrm{S}-42^{\circ} 30^{\prime} \mathrm{S}\right)$. Journal of South America Earth Sciences 1 (1): 75-88.

Schermer, E.R.; Cembrano, J.; Sanhueza, A.; McClelland, W.C. 1996. Geometry, kinematics and timing of intraarc shear, southern Chile. In International Geological Congress Proceedings 1: 214. Beijing.

SERNAGEOMIN, 2003. Mapa geológico de Chile, versión digital. Publicación Geológica Digital 4, CD-ROM, versión 1.0, 2003, base geológica escala $1: 1.000 .000$.

Silva, C.; Herrera, C.; Hervé, F. 2003. Petrogénesis de lavas y diques básicos de la Formación Traiguén, Región de

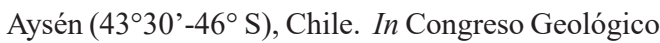
Chileno, No. 10, Actas (CD-ROM): 11 p. Concepción.

Somoza, R.; Ghidella, M.E. 2005. Convergencia en el margen occidental de América del Sur durante el Cenozoico: subducción de las placas de Nazca, Farallón y Aluk. Revista de la Asociación Geológica Argentina 60 (4): 797-809.

Somoza, R.; Ghidella, M.E. 2012. Late Cretaceous to recent plate motions in western South America revisited. Earth and Planetary Science Letters 331-332: 152-163. doi: 10.1016/j.epsl.2012.03.003.

Steffen, H. 1944. Patagonia Occidental. Las cordilleras patagónicas y sus regiones circundantes. Ediciones de la Universidad de Chile: 333 p. Santiago.

Tera, F.; Wasserburg, G.J. 1972. U-Th-Pb systematics in three Apollo 14 basalts and the problem of initial $\mathrm{Pb}$ in lunar rocks. Earth and Planetary Science Letters 14: 281-304.
Thiele, R.; Castillo, J.; Hein, R.; Ulloa, M. 1978. Geología del sector fronterizo de Chiloé Continental entre los $43^{\circ} 00^{\prime}$ y $43^{\circ} 45^{\prime}$ L.S., Chile. In Congreso Geológico Argentino, No 7, Resúmenes: 577-591. Neuquén.

Thomson, S.N.; Hervé, F.; Stöckhert, B. 2001. The Mesozoic-Cenozoic denudation history of the Patagonian Andes (southern Chile) and its correlation to different subduction processes. Tectonics 20: 693-711.

Thomson, S.N. 2002. Late Cenozoic geomorphic and tectonic evolution of the Patagonian Andes between latitudes $42^{\circ} \mathrm{S}$ and $46^{\circ} \mathrm{S}$ : an appraisal based on fission-track results from the transpressional intraarc Liquiñe-Ofqui fault zone. Geological Society of America Bulletin 114: 1159-1173.

Thomson, S.N.; Hervé, F. 2002. New time constraints for the age of metamorphism at the ancestral Pacific Gondwana margin of southern Chile. Revista Geológica de Chile 29 (2): 255-271. doi: 10.5027/ andgeoV29n2-a07.

Urbina, O. 2001. Geología de la cordillera norpatagónica en el área del río Palena, XI Región de Aysén, Chile. Undergraduate Thesis (Unpublished), Universidad de Chile, Departamento de Geología: 112 p.

Williams, I.S. 1998. U-Th-Pb geochronology by ion microprobe. In Applications of Micro-analytical Techniques to Understanding Mineralizing Processes (McKibben, M.A.; Shanks III, W.C.; Ridley, W.I.; editors). Reviews in economic geology. Society of Economic Geologists (SEG) 7: 1-35. doi: 10.5382/Rev.07.

Manuscript received: July 01, 2019; revised/accepted: February 26, 2020; available online: September 30, 2020. 